\title{
Unconditional Optimal Error Estimates for the Transient Navier-Stokes Equations with Damping
}

\author{
Minghao $\mathrm{Li}^{1}$, Zhenzhen $\mathrm{Li}^{2}$ and Dongyang $\mathrm{Shi}^{3, *}$ \\ ${ }^{1}$ College of Science, Henan University of Technology, Zhengzhou, Henan 450001, China \\ 2 College of Mathematics and Information Science, Zhengzhou University of Light \\ Industry, Zhengzhou, Henan 450002, China \\ ${ }^{3}$ School of Mathematics and Statistics, Zhengzhou University, Zhengzhou, Henan 450001, \\ China
}

Received 2 August 2020; Accepted (in revised version) 9 February 2021

\begin{abstract}
In this paper, the transient Navier-Stokes equations with damping are considered. Firstly, the semi-discrete scheme is discussed and optimal error estimates are derived. Secondly, a linearized backward Euler scheme is proposed. By the error split technique, the Stokes operator and the $H^{-1}$-norm estimate, unconditional optimal error estimates for the velocity in the norms $L^{\infty}\left(L^{2}\right)$ and $L^{\infty}\left(H^{1}\right)$, and the pressure in the norm $L^{\infty}\left(L^{2}\right)$ are deduced. Finally, two numerical examples are provided to confirm the theoretical analysis.
\end{abstract}

AMS subject classifications: 65N15, 65N30

Key words: Navier-Stokes equations with damping, linearized backward Euler scheme, error splitting technique, unconditional optimal error estimates.

\section{Introduction}

We consider the following transient Navier-Stokes equations with damping:

$$
\begin{cases}\mathbf{u}_{t}-v \Delta \mathbf{u}+(\mathbf{u} \cdot \nabla) \mathbf{u}+\alpha|\mathbf{u}|^{r-2} \mathbf{u}+\nabla p=\mathbf{f} & \text { in }(0, T] \times \Omega, \\ \operatorname{div} \mathbf{u}=0 & \text { in }(0, T] \times \Omega, \\ \mathbf{u}=0 & \text { on } \partial \Omega, \\ \mathbf{u}(0, \cdot)=\mathbf{u}_{0} & \text { in } \Omega,\end{cases}
$$

where $\Omega \subset \mathcal{R}^{2}$ is a convex polygon domain with the boundary $\partial \Omega, \mathbf{u}=\left(u_{1}, u_{2}\right)$ and $p$ are the fluid velocity and pressure, respectively, $\mathbf{f}$ is a given external force, $v$ is the viscosity coefficient, $2 \leq r<\infty$ and $\alpha$ are two damping parameters, respectively, and $|\cdot|$ is the

*Corresponding author.

Emails: lyminghao@126.com (M. Li), lizhenzhenpuyang@163.com (Z. Li), shi_dy@zzu.edu.cn (D. Shi) 
Euclidean norm. The damping comes from the resistance to the motion of the flow. It describes various physical phenomena such as porous media flow, drag or friction effects, and some dissipative mechanisms [1,2].

The existence and uniqueness of global weak and strong solutions for the problem (1.1) were analyzed in [3,4]. At the same time, some studies have been devoted to the numerical analysis of the stationary incompressible Stokes or Navier-Stokes equations with damping. In [5], the MAC finite difference scheme was presented for the Stokes equations with damping on non-uniform grids. In [6], the conforming mixed finite element method (MFEM) was developed, and the existence and uniqueness of the weak solutions were proved. In [7], the superclose and superconvergence phenomenon of some stable MFEs were studied. In [8-10], the local projection stabilized MFEMs with the $P_{1^{-}}$$P_{1}$ element pair were proposed for the Stokes or Navier-Stokes equations with damping. In $[11,12]$, the two-level and multi-level MFEMs were applied to the problem to save computation cost. In addition, the Navier-Stokes type variational inequality with nonlinear damping was also considered in [13]. However, there were few numerical methods reported for the transient Stokes or Navier-Stokes equations with damping.

On the other hand, in the finite element methods of the nonlinear problems, some time-step restrictions are usually required in the error estimates. In order to overcome this disadvantage, the error splitting technique was first presented in [14] for the nonlinear Joule heating equations and [15] for the incompressible miscible flow in porous media, respectively. Recently, this technique was applied to various nonlinear problems, such as the parabolic equation [16-18], the hyperbolic equation [19], the Schrödinger equation [20-24], the Landau-Lifshitz equation [25], the Ginzburg-Landau equations [26, 27], the Klein-Gordon-Schrödinger equations [28], the thermistor equations [29-31], the Navier-Stokes equations [32], the viscoelastic fluid flow equations [33], the MHD equations [34] and so on.

In this paper, we will research the transient Navier-Stokes equations with damping. We present the semi-discrete scheme for this problem, and derive optimal error estimates. Then we propose a linearized backward Euler scheme. Although unconditional optimal error estimates were obtained for the transient Navier-Stokes equations in [35, 36], the methods cannot be applied to the problem (1.1) for the nonlinear damping term may result in more complicated analysis, so we employ the error splitting technique in [32], which was used in the modified characteristics finite element methods of the NavierStokes equations. A time-discrete system is introduced, and the error is split into a temporal error and a spatial error. Then the temporal error and the regularity of the timediscrete system are presented. Subsequently, the space error and the boundedness of the velocity are derived. Finally, unconditional optimal error estimates are obtained. Especially, the analysis method of the pressure is different to that in [32]. The Stokes operator and the $H^{-1}$-norm estimate are employed, and consequently we obtained optimal error estimates for the pressure in the norm $L^{\infty}\left(L^{\infty}\right)$, while in [32], it is optimal in the norm $L^{\infty}\left(L^{2}\right)$.

Throughout this paper, we use the classical Sobolev spaces $W^{l, m}(\Omega), L^{l}(\Omega), H^{m}(\Omega)$ 
and $H_{0}^{m}(\Omega)$, where $1 \leq l \leq \infty$ and $m \geq 0$. Let $\|\cdot\|_{0, l}$ and $\|\cdot\|_{m}$ be the norms on $L^{l}(\Omega)$ and $H^{m}(\Omega)$, respectively, and $(\cdot, \cdot)$ be the natural inner product in $L^{2}(\Omega)$. We use $H^{-1}(\Omega)$ to denote the dual space of $H_{0}^{1}(\Omega)$, and $\langle\cdot, \cdot\rangle$ the duality product. In addition, we define the space $L^{l}(Y)$ with the norm

$$
\|\mathbf{u}\|_{L^{l}(Y)}=\left(\int_{0}^{T}\|\mathbf{u}(\cdot, t)\|_{Y}^{l} d t\right)^{\frac{1}{l}}
$$

and if $l=\infty$, the integral is replaced by the essential supremum. Finally, we denote by $C$ a generic positive constant, which may be different in different places, but independent of the mesh size $h$, the time step $\tau$ and the parameters $v, r, \alpha$.

\section{Mixed variational formulation}

Let

$$
V=\left(H_{0}^{1}(\Omega)\right)^{2}, \quad Q=L_{0}^{2}(\Omega)=\left\{q \in L^{2}(\Omega) \mid \int_{\Omega} q=0\right\},
$$

and define the skew-symmetric tri-linear form $b(\cdot ;, \cdot)$ on $V \times V \times V$ as

$$
\begin{aligned}
b(\mathbf{u} ; \mathbf{v}, \mathbf{w}) & =((\mathbf{u} \cdot \nabla) \mathbf{v}, \mathbf{w})+\frac{1}{2}((\operatorname{div} \mathbf{u}) \mathbf{v}, \mathbf{w}) \\
& =\frac{1}{2}((\mathbf{u} \cdot \nabla) \mathbf{v}, \mathbf{w})-\frac{1}{2}((\mathbf{u} \cdot \nabla) \mathbf{w}, \mathbf{v}) .
\end{aligned}
$$

Then the variational formulation of the problem (1.1) reads: for all $t \in(0, T]$, find $(\mathbf{u}, p) \in$ $V \times Q$ such that

$$
\begin{cases}\left(\mathbf{u}_{t}, \mathbf{v}\right)+v(\nabla \mathbf{u}, \nabla \mathbf{v})-(\operatorname{div} \mathbf{v}, p)+b(\mathbf{u} ; \mathbf{u}, \mathbf{v})+\alpha\left(|\mathbf{u}|^{r-2} \mathbf{u}, \mathbf{v}\right)=(\mathbf{f}, \mathbf{v}), & \forall \mathbf{u} \in V, \\ (\operatorname{div} \mathbf{u}, q)=0, & \forall q \in Q .\end{cases}
$$

Some estimates of the tri-linear form $b$ are given in the following lemma.

Lemma 2.1. The tri-linear form $b$ satisfies

$$
\begin{gathered}
|b(\mathbf{u} ; \mathbf{v}, \mathbf{w})| \leq C\|\nabla \mathbf{u}\|_{0}\|\nabla \mathbf{v}\|_{0}\|\nabla \mathbf{w}\|_{0}, \quad \forall \mathbf{u}, \mathbf{v}, \mathbf{w} \in\left(H_{0}^{1}(\Omega)\right)^{2}, \\
|b(\mathbf{u} ; \mathbf{v}, \mathbf{w})| \leq C\|\mathbf{u}\|_{0}\left(\|\nabla \mathbf{v}\|_{0,3}+\|\mathbf{v}\|_{0, \infty}\right)\|\nabla \mathbf{w}\|_{0} \\
\forall \mathbf{u}, \mathbf{w} \in\left(H_{0}^{1}(\Omega)\right)^{2}, \mathbf{v} \in\left(W^{1,3}(\Omega) \cap L^{\infty}(\Omega)\right)^{2} \\
|b(\mathbf{u} ; \mathbf{v}, \mathbf{w})| \leq C\left(\|\mathbf{u}\|_{0, \infty}+\|\nabla \mathbf{u}\|_{0,3}\right)\|\nabla \mathbf{v}\|_{0}\|\mathbf{w}\|_{0} \\
\forall \mathbf{v}, \mathbf{w} \in\left(H_{0}^{1}(\Omega)\right)^{2}, \mathbf{u} \in\left(W^{1,3}(\Omega) \cap L^{\infty}(\Omega)\right)^{2} \\
|b(\mathbf{u} ; \mathbf{v}, \mathbf{w})| \leq C\|\nabla \mathbf{u}\|_{0}\left(\|\nabla \mathbf{v}\|_{0,3}+\|\mathbf{v}\|_{0, \infty}\right)\|\mathbf{w}\|_{0} \\
\forall \mathbf{u}, \mathbf{w} \in\left(H_{0}^{1}(\Omega)\right)^{2}, \mathbf{v} \in\left(W^{1,3}(\Omega) \cap L^{\infty}(\Omega)\right)^{2} .
\end{gathered}
$$


Proof. Using (2.2) and Hölder inequality, it is easy to check that

$$
\begin{aligned}
|b(\mathbf{u} ; \mathbf{v}, \mathbf{w})| & =\left|\frac{1}{2}((\mathbf{u} \cdot \nabla) \mathbf{v}, \mathbf{w})-\frac{1}{2}((\mathbf{u} \cdot \nabla) \mathbf{w}, \mathbf{v})\right| \\
& \leq \frac{1}{2}\left(\|\mathbf{u}\|_{0}\|\nabla \mathbf{v}\|_{0,3}\|\mathbf{w}\|_{0,6}+\|\mathbf{u}\|_{0}\|\nabla \mathbf{w}\|_{0}\|\mathbf{v}\|_{0, \infty}\right) \\
& \leq C\|\mathbf{u}\|_{0}\left(\|\nabla \mathbf{v}\|_{0,3}+\|\mathbf{v}\|_{0, \infty}\right)\|\nabla \mathbf{w}\|_{0} \\
|b(\mathbf{u} ; \mathbf{v}, \mathbf{w})| & =\left|((\mathbf{u} \cdot \nabla) \mathbf{v}, \mathbf{w})+\frac{1}{2}((\operatorname{div} \mathbf{u}) \mathbf{v}, \mathbf{w})\right| \\
& \leq\|\mathbf{u}\|_{0, \infty}\|\nabla \mathbf{v}\|_{0}\|\mathbf{w}\|_{0}+\frac{1}{2}\|\operatorname{div} \mathbf{u}\|_{0,3}\|\mathbf{v}\|_{0,6}\|\mathbf{w}\|_{0} \\
& \leq C\left(\|\nabla \mathbf{u}\|_{0,3}+\|\mathbf{u}\|_{0, \infty}\right)\|\nabla \mathbf{v}\|_{0}\|\mathbf{w}\|_{0}
\end{aligned}
$$

which implies (2.4b) and (2.4c). The proof of (2.4d) is similar to that of (2.4c), and (2.4a) can be found in [35]. The proof is completed.

The following inequalities will be frequently used in the estimates of the nonlinear damping terms, which can be found in [37].

Lemma 2.2. For any $\mathbf{u}, \mathbf{v}, \mathbf{w} \in\left(L^{\infty}(\Omega)\right)^{2}$, we have

$$
\begin{aligned}
& \left(|\mathbf{u}|^{r-2} \mathbf{u}-|\mathbf{w}|^{r-2} \mathbf{w}, \mathbf{u}-\mathbf{w}\right) \geq 0, \\
& \left.|| \mathbf{u}\right|^{r-2} \mathbf{u}-|\mathbf{w}|^{r-2} \mathbf{w}\left|\leq M(|\mathbf{u}|+|\mathbf{w}|)^{r-2}\right| \mathbf{u}-\mathbf{w} \mid, \\
& \left|\left(|\mathbf{u}|^{r-2} \mathbf{u}-|\mathbf{w}|^{r-2} \mathbf{w}, \mathbf{v}\right)\right| \leq M\left(\|\mathbf{u}\|_{0, \infty}+\|\mathbf{w}\|_{0, \infty}\right)^{r-2}\|\mathbf{v}\|_{0}\|\mathbf{u}-\mathbf{w}\|_{0},
\end{aligned}
$$

where $M$ is dependent on $r$.

In addition, the steady Stokes equations

$$
\begin{cases}-v \Delta \mathbf{v}+\nabla q=\mathbf{g} & \text { in } \Omega, \\ \operatorname{div} \mathbf{v}=0 & \text { in } \Omega, \\ \mathbf{v}=0 & \text { on } \partial \Omega\end{cases}
$$

has a unique solution for the prescribed $\mathbf{g} \in\left(L^{2}(\Omega)\right)^{2}$, and satisfies [38]

$$
\|\mathbf{v}\|_{2}+\|q\|_{1} \leq C\|g\|_{0} .
$$

Finally, for the sake of simplicity, we assume that the problem (1.1) has a unique solution $(\mathbf{u}, p)$ with following regularity similar to that in [32]

$$
\begin{aligned}
& \left\|\mathbf{u}_{0}\right\|_{k}+\|\mathbf{u}\|_{L^{\infty}\left(H^{k+1}\right)}+\|p\|_{L^{\infty}\left(H^{k}\right)}+\left\|\mathbf{u}_{t}\right\|_{L^{\infty}\left(H^{k+1}\right)} \\
& \quad+\left\|p_{t}\right\|_{L^{\infty}\left(H^{k}\right)}+\left\|\mathbf{u}_{t t}\right\|_{L^{\infty}\left(L^{2}\right)} \leq K .
\end{aligned}
$$




\section{The semi-discrete scheme}

Let $\mathcal{T}_{h}$ be a uniformly regular partition of $\Omega$. The conforming finite element space pairs $V_{h} \subset V$ and $Q_{h} \subset Q$ are composed of piecewise polynomials of degrees at most $k$ and $k-1$ $(k \geq 1)$, respectively, which satisfy the inf-sup condition

$$
\sup _{\mathbf{v}_{h} \in V_{h}} \frac{\left(\operatorname{div} \mathbf{v}_{h}, q_{h}\right)}{\left\|\nabla \mathbf{v}_{h}\right\|_{0}} \geq \beta\left\|q_{h}\right\|_{0}
$$

where $\beta>0$ is independent of $h$.

Given $(\mathbf{u}, p) \in V \times Q$, the Stokes projection $\left(I_{h} \mathbf{u}, J_{h} p\right) \in V_{h} \times Q_{h}$ is defined to be the solution of

$$
\begin{cases}v\left(\nabla I_{h} \mathbf{u}, \nabla \mathbf{v}_{h}\right)+\left(\operatorname{div}_{h}, J_{h} p\right)=v\left(\nabla \mathbf{u}, \nabla \mathbf{v}_{h}\right)+\left(\operatorname{div}_{h}, p\right), & \forall \mathbf{v}_{h} \in V_{h}, \\ \left(\operatorname{div} I_{h} \mathbf{u}, q_{h}\right)=0, & \forall q_{h} \in Q_{h} .\end{cases}
$$

Then we have the following classic estimates $[39,40]$.

Lemma 3.1. Assume that $\mathbf{u} \in V \cap\left(H^{k+1}(\Omega)\right)^{2}, p \in Q \cap H^{k}(\Omega)$, then there hold

$$
\begin{aligned}
& v\left\|I_{h} \mathbf{u}\right\|_{0, \infty} \leq C\left(v\|\mathbf{u}\|_{2}+\|p\|_{1}\right), \\
& v\left\|\nabla I_{h} \mathbf{u}\right\|_{0, l} \leq C\left(v\|\nabla \mathbf{u}\|_{0, l}+\|p\|_{0, l}\right), \quad l=2,3 \\
& v\left(\left\|\mathbf{u}-I_{h} \mathbf{u}\right\|_{0}+h\left\|\nabla\left(\mathbf{u}-I_{h} \mathbf{u}\right)\right\|_{0}\right)+h\left\|p-J_{h} p\right\|_{0} \leq C h^{k+1}\left(v\|\mathbf{u}\|_{k+1}+\|p\|_{k}\right) \\
& v\left\|\mathbf{u}_{t}-I_{h} \mathbf{u}_{t}\right\|_{0} \leq C h^{k+1}\left(v\left\|\mathbf{u}_{t}\right\|_{k+1}+\left\|p_{t}\right\|_{k}\right) .
\end{aligned}
$$

The discrete Stokes operator $A_{h}: V_{h}^{\text {div }} \rightarrow V_{h}^{\text {div }}$ is defined by [41,42]

$$
\left(A_{h} \mathbf{v}_{h}, \mathbf{w}_{h}\right)=\left(A_{h}^{\frac{1}{2}} \mathbf{v}_{h}, A_{h}^{\frac{1}{2}} \mathbf{w}_{h}\right)=\left(\nabla \mathbf{v}_{h}, \nabla \mathbf{w}_{h}\right), \quad \forall \mathbf{v}_{h}, \mathbf{w}_{h} \in V_{h}^{\text {div }}
$$

where $V_{h}^{\operatorname{div}}=\left\{\mathbf{v}_{h} \in V_{h},\left(\operatorname{div} \mathbf{v}_{h}, q_{h}\right)=0, \forall q_{h} \in Q_{h}\right\}$.

Then we have the following properties of $A_{h}$.

Lemma 3.2. For any $\mathbf{v}_{h} \in V_{h}^{\text {div }}$, there hold

$$
\begin{aligned}
& \left\|\nabla A_{h}^{-1} \mathbf{v}_{h}\right\|_{0}=\left\|A_{h}^{-\frac{1}{2}} \mathbf{v}_{h}\right\|_{0}, \\
& \left\|\mathbf{v}_{h}\right\|_{-1} \leq C\left\|A_{h}^{-\frac{1}{2}} \mathbf{v}_{h}\right\|_{0} .
\end{aligned}
$$

Proof. From the definition of $A_{h}$, it is easy to obtain (3.5a), and (3.5b) can be found in [41, 42]. 
The semi-discrete scheme of the problem (1.1) reads: for all $t \in(0, T]$, find $\left(\mathbf{u}_{h}, p_{h}\right) \in$ $V_{h} \times Q_{h}$ such that

$$
\begin{cases}\left(\mathbf{u}_{h t}, \mathbf{v}_{h}\right)+v\left(\nabla \mathbf{u}_{h}, \nabla \mathbf{v}_{h}\right)+\alpha\left(\left|\mathbf{u}_{h}\right|^{r-2} \mathbf{u}_{h}, \mathbf{v}_{h}\right)-\left(\operatorname{div} \mathbf{v}_{h}, p_{h}\right) & \\ \quad+b\left(\mathbf{u}_{h} ; \mathbf{u}_{h}, \mathbf{v}_{h}\right)=\left(\mathbf{f}, \mathbf{v}_{h}\right), & \forall \mathbf{v}_{h} \in V_{h}, \\ \left(\operatorname{div} \mathbf{u}_{h}, q_{h}\right)=0, & \forall q_{h} \in Q_{h}, \\ \mathbf{u}_{h}(0)=I_{h} \mathbf{u}_{0} . & \end{cases}
$$

Let $\mathbf{e}_{h}=\mathbf{u}_{h}-I_{h} \mathbf{u}$, and subtract (3.6) from (2.3), then we obtain the following error equations

$$
\left\{\begin{array}{l}
\left(\mathbf{u}_{t}-I_{h} \mathbf{u}_{t}, \mathbf{v}_{h}\right)-\left(\mathbf{e}_{h t}, \mathbf{v}_{h}\right)-v\left(\nabla \mathbf{e}_{h}, \nabla \mathbf{v}_{h}\right)-\left(J_{h} p-p_{h}, \operatorname{div}_{h}\right) \\
\quad+b\left(\mathbf{u}-I_{h} \mathbf{u} ; \mathbf{u}, \mathbf{v}_{h}\right)+b\left(I_{h} \mathbf{u} ; \mathbf{u}-I_{h} \mathbf{u}, \mathbf{v}_{h}\right)-b\left(\mathbf{e}_{h} ; I_{h} \mathbf{u}, \mathbf{v}_{h}\right)-b\left(\mathbf{u}_{h} ; \mathbf{e}_{h}, \mathbf{v}_{h}\right) \\
\quad+\alpha\left(|\mathbf{u}|^{r-2} \mathbf{u}-\left|I_{h} \mathbf{u}\right|^{r-2} I_{h} \mathbf{u}, \mathbf{v}_{h}\right)+\alpha\left(\left|I_{h} \mathbf{u}\right|^{r-2} I_{h} \mathbf{u}-\left|\mathbf{u}_{h}\right|^{r-2} \mathbf{u}_{h}, \mathbf{v}_{h}\right)=0 \\
\left(\operatorname{div} \mathbf{e}_{h}, q_{h}\right)=0 .
\end{array}\right.
$$

Now we turn to optimal error estimates for the velocity $\mathbf{u}$.

Theorem 3.1. Let $(\mathbf{u}, p)$ and $\left(\mathbf{u}_{h}, p_{h}\right)$ be the solutions of (1.1) and (3.6). Assume that the solution $(\mathbf{u}, p)$ satisfies the regularity condition (2.9). Then it holds for all $t \in(0, T]$ that

$$
\left\|\mathbf{e}_{h}\right\|_{0}+h\left\|\nabla \mathbf{e}_{h}\right\|_{0} \leq C L_{1} h^{k+1},
$$

where

$$
L_{1}^{2}:=e^{C \frac{K^{2}}{v^{3}}}\left(\frac{K^{2}}{v^{3}}+\alpha^{2} M^{2} \frac{K^{2 r-2}}{v^{2 r-1}}+\frac{K^{4}}{v^{3}}+\frac{K^{4}}{v^{5}}\right) .
$$

Moreover, assume that $h$ is small enough, we have

$$
\begin{aligned}
\left\|\nabla \mathbf{u}_{h}\right\|_{0} \leq C\left(1+\frac{K}{v}\right), \\
\left\|\mathbf{u}_{h}\right\|_{0, \infty} \leq C\left(1+\frac{K}{v}\right) .
\end{aligned}
$$

Proof. Take $\mathbf{v}_{h}=\mathbf{e}_{h} \in V_{h}^{\text {div }}$ in (3.7), and note that

$$
\left(\left|\mathbf{u}_{h}\right|^{r-2} \mathbf{u}_{h}-\left|I_{h} \mathbf{u}\right|^{r-2} I_{h} \mathbf{u}, \mathbf{e}_{h}\right) \geq 0,
$$

then it gives

$$
\begin{aligned}
& \frac{1}{2} \frac{d}{d t}\left\|\mathbf{e}_{h}\right\|_{0}^{2}+v\left\|\nabla \mathbf{e}_{h}\right\|_{0}^{2} \leq\left(\mathbf{u}_{t}-I_{h} \mathbf{u}_{t}, \mathbf{e}_{h}\right)+\alpha\left(|\mathbf{u}|^{r-2} \mathbf{u}-\left|I_{h} \mathbf{u}\right|^{r-2} I_{h} \mathbf{u}, \mathbf{e}_{h}\right) \\
& +b\left(\mathbf{u}-I_{h} \mathbf{u} ; \mathbf{u}, \mathbf{e}_{h}\right)+b\left(I_{h} \mathbf{u} ; \mathbf{u}-I_{h} \mathbf{u}, \mathbf{e}_{h}\right)-b\left(\mathbf{e}_{h} ; I_{h} \mathbf{u}, \mathbf{e}_{h}\right): \triangleq \sum_{i=1}^{5} E_{i}
\end{aligned}
$$


Next we estimate all terms on the right hand-side of (3.11). It is easy to check that

$$
\left|E_{1}\right| \leq C\left\|\mathbf{u}_{t}-I_{h} \mathbf{u}_{t}\right\|_{0}\left\|\nabla \mathbf{e}_{h}\right\|_{0} \leq C \frac{K^{2}}{v^{3}} h^{2(k+1)}+\frac{v}{6}\left\|\nabla \mathbf{e}_{h}\right\|_{0}^{2} .
$$

By Lemma 2.2 and Lemma 3.1, the nonlinear damping term can be estimated as

$$
\begin{aligned}
\left|E_{2}\right| & \leq \alpha M\left\|\mathbf{u}-I_{h} \mathbf{u}\right\|_{0}\left(\|\mathbf{u}\|_{0, \infty}+\left\|I_{h} \mathbf{u}\right\|_{0, \infty}\right)^{r-2}\left\|\mathbf{e}_{h}\right\|_{0} \\
& \leq C \alpha^{2} M^{2} \frac{K^{2 r-2}}{v^{2 r-1}} h^{2(k+1)}+\frac{v}{6}\left\|\nabla \mathbf{e}_{h}\right\|_{0}^{2} .
\end{aligned}
$$

According to Lemma 2.1 and Lemma 3.1, we obtain

$$
\begin{aligned}
\left|E_{3}\right| & \leq C\left\|\mathbf{u}-I_{h} \mathbf{u}\right\|_{0}\left(\|\nabla \mathbf{u}\|_{0,3}+\|\mathbf{u}\|_{0, \infty}\right)\left\|\nabla \mathbf{e}_{h}\right\|_{0} \leq C \frac{K^{4}}{v^{3}} h^{2 k+2}+\frac{v}{6}\left\|\nabla \mathbf{e}_{h}\right\|_{0}^{2}, \\
\left|E_{4}\right| & =\left|b\left(I_{h} \mathbf{u} ; \mathbf{e}_{h}, \mathbf{u}-I_{h} \mathbf{u}\right)\right| \leq C\left(\left\|I_{h} \mathbf{u}\right\|_{0, \infty}+\left\|\nabla I_{h} \mathbf{u}\right\|_{0,3}\right)\left\|\nabla \mathbf{e}_{h}\right\|_{0}\left\|\mathbf{u}-I_{h} \mathbf{u}\right\|_{0} \\
& \leq C \frac{K^{4}}{v^{5}} h^{2 k+2}+\frac{v}{6}\left\|\nabla \mathbf{e}_{h}\right\|_{0}^{2}, \\
\left|E_{5}\right| & \leq C\left\|\mathbf{e}_{h}\right\|_{0}\left(\left\|\nabla I_{h} \mathbf{u}\right\|_{0,3}+\left\|I_{h} \mathbf{u}\right\|_{0, \infty}\right)\left\|\nabla \mathbf{e}_{h}\right\|_{0} \leq C \frac{K^{2}}{v^{3}}\left\|\mathbf{e}_{h}\right\|_{0}^{2}+\frac{v}{6}\left\|\nabla \mathbf{e}_{h}\right\|_{0}^{2} .
\end{aligned}
$$

Inserting (3.12)-(3.14c) into (3.11) yields

$$
\begin{aligned}
& \frac{1}{2} \frac{d}{d t}\left\|\mathbf{e}_{h}\right\|_{0}^{2}+\frac{v}{6}\left\|\nabla \mathbf{e}_{h}\right\|_{0}^{2} \\
\leq & C\left(\frac{K^{2}}{v^{3}}+\alpha^{2} M^{2} \frac{K^{2 r-2}}{v^{2 r-1}}+\frac{K^{4}}{v^{3}}+\frac{K^{4}}{v^{5}}\right) h^{2 k+2}+C \frac{K^{2}}{v^{3}}\left\|\mathbf{e}_{h}\right\|_{0}^{2} .
\end{aligned}
$$

By Gronwall lemma, and note that $\mathbf{e}_{h}(\cdot, 0)=0$, we deduce

$$
\left\|\mathbf{e}_{h}\right\|_{0}^{2}+\int_{0}^{T} v\left\|\nabla \mathbf{e}_{h}\right\|_{0}^{2} d t \leq C L_{1}^{2} h^{2 k+2},
$$

which together with the inverse inequality implies

$$
\left\|\nabla \mathbf{e}_{h}\right\|_{0} \leq C h^{-1}\left\|\mathbf{e}_{h}\right\|_{0} \leq C L_{1} h^{k} .
$$

In addition, assume that $h$ is small enough, which ensures $L_{1} h^{k} \leq 1$, then we have

$$
\begin{aligned}
& \left\|\nabla \mathbf{u}_{h}\right\|_{0} \leq\left\|\nabla \mathbf{e}_{h}\right\|_{0}+\left\|\nabla I_{h} \mathbf{u}\right\|_{0} \leq C\left(1+\frac{K}{v}\right), \\
& \left\|\mathbf{u}_{h}\right\|_{0, \infty} \leq\left\|\mathbf{e}_{h}\right\|_{0, \infty}+\left\|I_{h} \mathbf{u}\right\|_{0, \infty} \leq C\left(h^{-1}\left\|\mathbf{e}_{h}\right\|_{0}+\left\|I_{h} \mathbf{u}\right\|_{0, \infty}\right) \leq C\left(1+\frac{K}{v}\right) .
\end{aligned}
$$

This completed the proof. 
Next we derive the error estimate of the pressure. To arrive at this, we first bound the term $\left\|\mathbf{e}_{h t}\right\|_{-1}$, where the $H^{-1}$-norm $\|\cdot\|_{-1}$ is defined by

$$
\|\phi\|_{-1}=\sup _{\varphi \in H_{0}^{1}(\Omega)} \frac{\langle\phi, \varphi\rangle}{\|\nabla \varphi\|_{0}}, \quad \forall \phi \in H^{-1}(\Omega) .
$$

Lemma 3.3. Under the conditions of Theorem 3.1, for all $t \in(0, T]$, we have

$$
\left\|\mathbf{e}_{h t}\right\|_{-1} \leq C L_{2} h^{k}
$$

where

$$
L_{2}:=\frac{K}{v}+\frac{K^{2}}{v}+\frac{K^{2}}{v^{2}}+\left(1+\frac{K}{v}\right) L_{1}+\alpha M\left(\frac{K^{r-1}}{v^{r-1}}+\left(1+\frac{K}{v}\right)^{r-2} L_{1}\right) .
$$

Proof. Setting $\mathbf{v}_{h}=A_{h}^{-1} \mathbf{e}_{h t} \in V_{h}^{\text {div }}$ in (3.7) leads to

$$
\begin{aligned}
& \left\|A_{h}^{-\frac{1}{2}} \mathbf{e}_{h t}\right\|_{0}^{2}=\left(\mathbf{e}_{h t}, A_{h}^{-1} \mathbf{e}_{h t}\right) \\
& =\left(\mathbf{u}_{t}-I_{h} \mathbf{u}_{t}, A_{h}^{-1} \mathbf{e}_{h t}\right)-v\left(\nabla \mathbf{e}_{h}, \nabla A_{h}^{-1} \mathbf{e}_{h t}\right) \\
& \quad+\alpha\left(|\mathbf{u}|^{r-2} \mathbf{u}-\left|I_{h} \mathbf{u}\right|^{r-2} I_{h} \mathbf{u}, A_{h}^{-1} \mathbf{e}_{h t}\right) \\
& \quad+\alpha\left(\left|I_{h} \mathbf{u}\right|^{r-2} I_{h} \mathbf{u}-\left|\mathbf{u}_{h}\right|^{r-2} \mathbf{u}_{h}, A_{h}^{-1} \mathbf{e}_{h t}\right) \\
& \quad+b\left(\mathbf{u}-I_{h} \mathbf{u} ; \mathbf{u}, A_{h}^{-1} \mathbf{e}_{h t}\right)+b\left(I_{h} \mathbf{u} ; \mathbf{u}-I_{h} \mathbf{u}, A_{h}^{-1} \mathbf{e}_{h t}\right) \\
& \quad-b\left(\mathbf{e}_{h} ; I_{h} \mathbf{u}, A_{h}^{-1} \mathbf{e}_{h t}\right)-b\left(\mathbf{u}_{h} ; \mathbf{e}_{h}, A_{h}^{-1} \mathbf{e}_{h t}\right) \stackrel{\Delta}{:}=\sum_{i=1}^{8} H_{i} .
\end{aligned}
$$

Since $A_{h}^{-1} \mathbf{e}_{h t} \in\left(H_{0}^{1}(\Omega)\right)^{2}$, by Poincaré inequality and (3.5a), we have

$$
\left\|A_{h}^{-1} \mathbf{e}_{h t}\right\|_{0} \leq\left\|A_{h}^{-1} \mathbf{e}_{h t}\right\|_{1} \leq C\left\|\nabla A_{h}^{-1} \mathbf{e}_{h t}\right\|_{0}=C\left\|A_{h}^{-\frac{1}{2}} \mathbf{e}_{h t}\right\|_{0}
$$

Then we estimate the above eight terms. In fact, from (3.3c), (3.5a), (3.8) and (3.22), it is easy to see that

$$
\begin{aligned}
& \left|H_{1}\right| \leq\left\|\mathbf{u}_{t}-I_{h} \mathbf{u}_{t}\right\|_{0}\left\|A_{h}^{-1} \mathbf{e}_{h t}\right\|_{0} \leq C \frac{K}{v} h^{k+1}\left\|A_{h}^{-\frac{1}{2}} \mathbf{e}_{h t}\right\|_{0} \\
& \left|H_{2}\right| \leq v\left\|\nabla \mathbf{e}_{h}\right\|_{0}\left\|\nabla A_{h}^{-1} \mathbf{e}_{h t}\right\|_{0} \leq C L_{1} h^{k}\left\|A_{h}^{-\frac{1}{2}} \mathbf{e}_{h t}\right\|_{0} .
\end{aligned}
$$

In view of (2.6c), (3.18b), (3.22) and Lemma 3.1, it follows for the nonlinear damping 
terms that

$$
\begin{aligned}
\left|H_{3}\right| & \leq \alpha M\left\|\mathbf{u}-I_{h} \mathbf{u}\right\|_{0}\left(\|\mathbf{u}\|_{0, \infty}+\left\|I_{h} \mathbf{u}\right\|_{0, \infty}\right)^{r-2}\left\|A_{h}^{-1} \mathbf{e}_{h t}\right\|_{0} \\
& \leq C \alpha M \frac{K^{r-1}}{v^{r-1}} h^{k+1}\left\|A_{h}^{-\frac{1}{2}} \mathbf{e}_{h t}\right\|_{0} \\
\left|H_{4}\right| & \leq \alpha M\left\|\mathbf{e}_{h}\right\|_{0}\left(\left\|\mathbf{u}_{h}\right\|_{0, \infty}+\left\|I_{h} \mathbf{u}\right\|_{0, \infty}\right)^{r-2}\left\|A_{h}^{-1} \mathbf{e}_{h t}\right\|_{0} \\
& \leq C \alpha M\left(1+\frac{K}{v}\right)^{r-2} L_{1} h^{k+1}\left\|A_{h}^{-\frac{1}{2}} \mathbf{e}_{h t}\right\|_{0}
\end{aligned}
$$

Using (2.4a), (3.5a), (3.18a) and Lemma 3.1, the tri-linear terms can be bounded as

$$
\begin{aligned}
& \left|H_{5}\right| \leq C\left\|\nabla\left(\mathbf{u}-I_{h} \mathbf{u}\right)\right\|_{0}\|\nabla \mathbf{u}\|_{0}\left\|\nabla A_{h}^{-1} \mathbf{e}_{h t}\right\|_{0} \leq C \frac{K^{2}}{v} h^{k}\left\|A_{h}^{-\frac{1}{2}} \mathbf{e}_{h t}\right\|_{0} \\
& \left|H_{6}\right| \leq C\left\|\nabla\left(\mathbf{u}-I_{h} \mathbf{u}\right)\right\|_{0}\left\|\nabla I_{h} \mathbf{u}\right\|_{0}\left\|\nabla A_{h}^{-1} \mathbf{e}_{h t}\right\|_{0} \leq C \frac{K^{2}}{v^{2}} h^{k}\left\|A_{h}^{-\frac{1}{2}} \mathbf{e}_{h t}\right\|_{0} \\
& \left|H_{7}\right| \leq C\left\|\nabla \mathbf{e}_{h}\right\|_{0}\left\|\nabla I_{h} \mathbf{u}\right\|_{0}\left\|\nabla A_{h}^{-1} \mathbf{e}_{h t}\right\|_{0} \leq C \frac{K}{v} L_{1} h^{k}\left\|A_{h}^{-\frac{1}{2}} \mathbf{e}_{h t}\right\|_{0}^{\prime} \\
& \left|H_{8}\right| \leq C\left\|\nabla \mathbf{u}_{h}\right\|_{0}\left\|\nabla \mathbf{e}_{h}\right\|_{0}\left\|\nabla A_{h}^{-1} \mathbf{e}_{h t}\right\|_{0} \leq C\left(1+\frac{K}{v}\right) L_{1} h^{k}\left\|A_{h}^{-\frac{1}{2}} \mathbf{e}_{h t}\right\|_{0} .
\end{aligned}
$$

Inserting the estimates (3.23a), (3.23b), (3.24a)-(3.25d) into (3.21) yields

$$
\left\|A_{h}^{-\frac{1}{2}} \mathbf{e}_{h t}\right\|_{0} \leq C L_{2} h^{k}
$$

In addition, from (3.5b), we have

$$
\left\|\mathbf{e}_{h t}\right\|_{-1} \leq C\left\|A_{h}^{-\frac{1}{2}} \mathbf{e}_{h t}\right\|_{0}^{\prime}
$$

which together with (3.26) yields the estimate (3.20).

Then based on Lemma 3.3, we have the error estimate for the pressure.

Theorem 3.2. Under the conditions of Theorem 3.1, the following error estimate holds for all $t \in(0, T]$

$$
\left\|p_{h}-J_{h} p\right\|_{0} \leq C L_{2} h^{k}
$$

Proof. Rearrange the error equation (3.7), it gives

$$
\begin{aligned}
& \left(J_{h} p-p_{h}, \operatorname{div} \mathbf{v}_{h}\right) \\
= & \left(\mathbf{u}_{t}-I_{h} \mathbf{u}_{t}, \mathbf{v}_{h}\right)-\left(\mathbf{e}_{h t}, \mathbf{v}_{h}\right)-v\left(\nabla \mathbf{e}_{h}, \nabla \mathbf{v}_{h}\right) \\
& +\alpha\left(|\mathbf{u}|^{r-2} \mathbf{u}-\left|I_{h} \mathbf{u}\right|^{r-2} I_{h} \mathbf{u}, \mathbf{v}_{h}\right)+\alpha\left(\left|I_{h} \mathbf{u}\right|^{r-2} I_{h} \mathbf{u}-\left|\mathbf{u}_{h}\right|^{r-2} \mathbf{u}_{h}, \mathbf{v}_{h}\right) \\
& +b\left(\mathbf{u}-I_{h} \mathbf{u} ; \mathbf{u}, \mathbf{v}_{h}\right)+b\left(I_{h} \mathbf{u} ; \mathbf{u}-I_{h} \mathbf{u}, \mathbf{v}_{h}\right)-b\left(\mathbf{e}_{h} ; I_{h} \mathbf{u}, \mathbf{v}_{h}\right)-b\left(\mathbf{u}_{h} ; \mathbf{e}_{h}, \mathbf{v}_{h}\right) .
\end{aligned}
$$


The above terms can be estimated similarly as that in Lemma 3.3, and then using the inf-sup condition, we obtain (3.28).

Finally, by Theorem 3.1, Theorem 3.2 and the triangle inequality, we derive the following optimal error estimates for the problem (3.6).

Theorem 3.3. Under the conditions of Theorem 3.1, for all $t \in(0, T]$, we have

$$
\begin{aligned}
& \left\|\mathbf{u}-\mathbf{u}_{h}\right\|_{0}+h\left\|\nabla\left(\mathbf{u}-\mathbf{u}_{h}\right)\right\|_{0} \leq C L_{1} h^{k+1}, \\
& \left\|p-p_{h}\right\|_{0} \leq C L_{2} h^{k} .
\end{aligned}
$$

\section{A linearized backward Euler scheme}

In this section, we consider the time discretization of the mixed finite element scheme (3.6). Let $0=t_{0}<t_{1}<\cdots<t_{N}=T$ be a uniform partition of the time interval $[0, T]$ with $t_{n}=n \tau$, and $N$ being a positive integer. Set $g^{n}=g\left(\cdot, t_{n}\right)$, and for the sequence of functions $\left\{g^{n}\right\}_{n=0}^{N}$, we define the discrete time derivative

$$
D_{\tau} g^{n}=\left(g^{n}-g^{n-1}\right) / \tau .
$$

With above notations, a linearized backward Euler scheme of (3.6) reads: for $n=1,2, \cdots, N$, given $\mathbf{f}^{n} \in V^{\prime}$ and $\mathbf{U}_{h}^{n-1} \in V_{h}$, find $\left(\mathbf{U}_{h}^{n}, P_{h}^{n}\right) \in V_{h} \times Q_{h}$ satisfying

$$
\begin{cases}\left(D_{\tau} \mathbf{U}_{h}^{n}, \mathbf{v}_{h}\right)+v\left(\nabla \mathbf{U}_{h}^{n}, \nabla \mathbf{v}_{h}\right)-\left(\operatorname{div}_{h}, P_{h}^{n}\right)+b\left(\mathbf{U}_{h}^{n-1} ; \mathbf{U}_{h}^{n}, \mathbf{v}_{h}\right) & \\ \quad+\alpha\left(\left|\mathbf{U}_{h}^{n-1}\right|^{r-2} \mathbf{U}_{h}^{n-1}, \mathbf{v}_{h}\right)=\left(\mathbf{f}^{n}, \mathbf{v}_{h}\right), & \forall \mathbf{v}_{h} \in V_{h}, \\ \left(\operatorname{div} \mathbf{U}_{h}^{n}, q_{h}\right)=0, & \forall q_{h} \in Q_{h}, \\ \mathbf{U}_{h}^{0}=I_{h} \mathbf{u}_{0} . & \end{cases}
$$

\subsection{The time discrete system}

To obtain the bounds of $\mathbf{U}_{h}^{n}$, we introduce the following time discrete system

$$
\begin{cases}D_{\tau} \mathbf{U}^{n}-v \Delta \mathbf{U}^{n}+\left(\mathbf{U}^{n-1} \cdot \nabla\right) \mathbf{U}^{n}+\alpha\left|\mathbf{U}^{n-1}\right|^{r-2} \mathbf{U}^{n-1}+\nabla P^{n}=\mathbf{f}^{n} & \text { in } \Omega, \\ \operatorname{div} \mathbf{U}^{n}=0 & \text { in } \Omega, \\ \mathbf{U}^{n}=0 & \text { on } \partial \Omega, \\ \mathbf{U}^{0}=\mathbf{u}_{0} & \text { in } \Omega .\end{cases}
$$

For each given time level $n,(4.3)$ is the Oseen equations, and if $\mathbf{U}^{n-1} \in\left(L^{\infty}(\Omega)\right)^{2}$, it has a unique solution [40]. 
Let $\mathbf{e}^{n}=\mathbf{u}^{n}-\mathbf{U}^{n}$, and subtract (4.3) from (1.1) with $t=t_{n}$, then we have the following error equations

$$
\begin{cases}D_{\tau} \mathbf{e}^{n}-v \Delta \mathbf{e}^{n}+\Lambda_{1}{ }^{n}+\Lambda_{2}^{n}+\nabla\left(p^{n}-P^{n}\right)=\mathbf{R}_{1}^{n}+\mathbf{R}_{2}^{n}+\mathbf{R}_{3}^{n} & \text { in } \Omega, \\ \operatorname{div} \mathbf{e}^{n}=0 & \text { in } \Omega, \\ \mathbf{e}^{n}=0 & \text { on } \partial \Omega, \\ \mathbf{e}^{0}=0 & \text { in } \Omega,\end{cases}
$$

where

$$
\begin{aligned}
& \boldsymbol{\Lambda}_{1}^{n}=\left(\mathbf{u}^{n-1} \cdot \nabla\right) \mathbf{u}^{n}-\left(\mathbf{U}^{n-1} \cdot \nabla\right) \mathbf{U}^{n}, \\
& \boldsymbol{\Lambda}_{2}^{n}=\alpha\left|\mathbf{u}^{n-1}\right|^{r-2} \mathbf{u}^{n-1}-\alpha\left|\mathbf{U}^{n-1}\right|^{r-2} \mathbf{U}^{n-1}, \\
& \mathbf{R}_{1}^{n}=D_{\tau} \mathbf{u}^{n}-\mathbf{u}_{t}^{n}, \quad \mathbf{R}_{2}^{n}=\left(\mathbf{u}^{n-1} \cdot \nabla\right) \mathbf{u}^{n}-\left(\mathbf{u}^{n} \cdot \nabla\right) \mathbf{u}^{n}, \\
& \mathbf{R}_{3}^{n}=\alpha\left|\mathbf{u}^{n-1}\right|^{r-2} \mathbf{u}^{n-1}-\alpha\left|\mathbf{u}^{n}\right|^{r-2} \mathbf{u}^{n} .
\end{aligned}
$$

Now we derive the temporal error estimate and the regularity of the time discrete system (4.3).

Lemma 4.1. Let $(\mathbf{u}, p)$ and $\left(\mathbf{U}^{n}, P^{n}\right)$ be the solutions of the problems (1.1) and (4.3), respectively. Assume that $(\mathbf{u}, p)$ satisfies the regularity condition (2.9), and the time step $\tau$ is small enough. Then we have

$$
\begin{aligned}
& \max _{0 \leq n \leq N}\left\|\mathbf{e}^{n}\right\|_{2} \leq 1, \\
& \max _{1 \leq n \leq N}\left(\left\|\mathbf{e}^{n}\right\|_{0}+\left\|\nabla \mathbf{e}^{n}\right\|_{0}\right) \leq \frac{C}{\sqrt{v}} L_{3} \tau, \\
& \max _{1 \leq n \leq N}\left(\left\|\mathbf{U}^{n}\right\|_{2}+\left\|P^{n}\right\|_{1}\right) \leq 1+K, \\
& \tau \sum_{n=1}^{N}\left(\left\|D_{\tau} \mathbf{U}^{n}\right\|_{2}^{2}+\left\|D_{\tau} P^{n}\right\|_{1}^{2}\right) \leq C K_{0}^{2},
\end{aligned}
$$

where

$$
\begin{aligned}
& L_{3}^{2}:=e^{\frac{C}{v}\left(1+K^{2}+\alpha^{2} M^{2}(K+1)^{2 r-4}\right)\left(K^{2}+K^{4}+\alpha^{2} M^{2} K^{2 r-2}\right),} \\
& K_{0}:=K+K^{2}+\alpha M K^{r-1}+\left(1+K+\alpha M(1+K)^{r-2}\right) \frac{L_{3}}{\sqrt{v}} .
\end{aligned}
$$

Proof. We prove the following inequality

$$
\left\|\mathbf{e}^{m}\right\|_{2} \leq 1
$$

by mathematical induction for all $m=0,1, \cdots, N$. 
Since $\mathbf{e}^{0}=0$, (4.8) holds for $m=0$.

We assume that (4.8) holds for $m \leq n-1$, then it follows that

$$
\left\|\mathbf{U}^{m}\right\|_{0, \infty}=\left\|\mathbf{u}^{m}\right\|_{0, \infty}+\left\|\mathbf{e}^{m}\right\|_{0, \infty} \leq\|\mathbf{u}\|_{L^{\infty}\left(H^{2}\right)}+\left\|\mathbf{e}^{m}\right\|_{2} \leq K+1 .
$$

When $m=n$, multiply the first formula of (4.4) both side by $D_{\tau} \mathbf{e}^{n}$ and integrate it over $\Omega$, we get

$$
\begin{aligned}
& \left\|D_{\tau} \mathbf{e}^{n}\right\|_{0}^{2}+\frac{v}{2 \tau}\left(\left\|\nabla \mathbf{e}^{n}\right\|_{0}^{2}+\left\|\nabla\left(\mathbf{e}^{n}-\mathbf{e}^{n-1}\right)\right\|_{0}^{2}-\left\|\nabla \mathbf{e}^{n-1}\right\|_{0}^{2}\right) \\
= & -\left(\boldsymbol{\Lambda}_{1}^{n}+\boldsymbol{\Lambda}_{2}^{n}, D_{\tau} \mathbf{e}^{n}\right)+\left(\mathbf{R}_{1}^{n}+\mathbf{R}_{2}^{n}+\mathbf{R}_{3}^{n}, D_{\tau} \mathbf{e}^{n}\right) .
\end{aligned}
$$

By Taylor formula, we can easily get

$$
\begin{gathered}
\left\|\mathbf{u}_{t}^{n}-D_{\tau} \mathbf{u}^{n}\right\|_{0} \leq C \tau\left\|\mathbf{u}_{t t}\right\|_{L^{\infty}\left(L^{2}\right)}, \\
\left\|\mathbf{u}^{n}-\mathbf{u}^{n-1}\right\|_{0} \leq C \tau\left\|\mathbf{u}_{t}\right\|_{L^{\infty}\left(L^{2}\right)}
\end{gathered}
$$

which together with (2.6b) yields

$$
\left\|\mathbf{R}_{1}^{n}\right\|_{0}+\left\|\mathbf{R}_{2}^{n}\right\|_{0}+\left\|\mathbf{R}_{3}^{n}\right\|_{0} \leq C\left(K+K^{2}+\alpha M K^{r-1}\right) \tau .
$$

Again using (2.6b) and (4.9), we find that

$$
\begin{aligned}
\left\|\boldsymbol{\Lambda}_{1}^{n}\right\|_{0} & \leq\left\|\mathbf{e}^{n-1}\right\|_{0,3}\left\|\nabla \mathbf{u}^{n}\right\|_{0,6}+\left\|\mathbf{U}^{n-1}\right\|_{0, \infty}\left\|\nabla \mathbf{e}^{n}\right\|_{0} \\
& \leq C(K+1)\left(\left\|\nabla \mathbf{e}^{n-1}\right\|_{0}+\left\|\nabla \mathbf{e}^{n}\right\|_{0}\right), \\
\left\|\boldsymbol{\Lambda}_{2}^{n}\right\|_{0} & \leq \alpha M\left(\left\|\mathbf{u}^{n-1}\right\|_{0, \infty}+\left\|\mathbf{U}^{n-1}\right\|_{0, \infty}\right)^{r-2}\left\|\mathbf{e}^{n-1}\right\|_{0} \\
& \leq C \alpha M(K+1)^{r-2}\left\|\nabla \mathbf{e}^{n-1}\right\|_{0} .
\end{aligned}
$$

With (4.12)-(4.13b) and Young inequality, (4.10) reduces to

$$
\begin{aligned}
& \frac{1}{6}\left\|D_{\tau} \mathbf{e}^{n}\right\|_{0}^{2}+\frac{v}{2 \tau}\left\|\nabla \mathbf{e}^{n}\right\|_{0}^{2} \\
\leq & \frac{v}{2 \tau}\left\|\nabla \mathbf{e}^{n-1}\right\|_{0}^{2}+C\left(K^{2}+K^{4}+\alpha^{2} M^{2} K^{2 r-2}\right) \tau^{2} \\
& +C\left(1+K^{2}+\alpha^{2} M^{2}(K+1)^{2 r-4}\right)\left(\left\|\nabla \mathbf{e}^{n-1}\right\|_{0}^{2}+\left\|\nabla \mathbf{e}^{n}\right\|_{0}^{2}\right) .
\end{aligned}
$$

Summing (4.14) from 1 to $n$ and multiplying by $\tau$, we get

$$
\begin{aligned}
& v\left\|\nabla \mathbf{e}^{n}\right\|_{0}^{2}+\tau \sum_{m=1}^{n}\left\|D_{\tau} \mathbf{e}^{m}\right\|_{0}^{2} \\
\leq & C\left(K^{2}+K^{4}+\alpha^{2} M^{2} K^{2 r-2}\right) \tau^{2}+\frac{C}{v}\left(1+K^{2}+\alpha^{2} M^{2}(K+1)^{2 r-4}\right) \tau \sum_{m=1}^{n} v\left\|\nabla \mathbf{e}^{m}\right\|_{0}^{2} .
\end{aligned}
$$


By Gronwall lemma, there exists a positive constant $\tau_{1}$, such that for $\tau<\tau_{1}$, it holds

$$
v\left\|\nabla \mathbf{e}^{n}\right\|_{0}^{2}+\tau \sum_{m=1}^{n}\left\|D_{\tau} \mathbf{e}^{m}\right\|_{0}^{2} \leq C L_{3}^{2} \tau^{2}
$$

which also implies

$$
\left\|\mathbf{e}^{n}\right\|_{0} \leq C\left\|\nabla \mathbf{e}^{n}\right\|_{0} \leq \frac{C}{\sqrt{v}} L_{3} \tau
$$

Next, we consider the following equations

$$
\begin{cases}-v \Delta \mathbf{e}^{n}+\nabla\left(p^{n}-P^{n}\right)=-D_{\tau} \mathbf{e}^{n}-\Lambda_{1}^{n}-\Lambda_{2}^{n}+\mathbf{R}_{1}^{n}+\mathbf{R}_{2}^{n}+\mathbf{R}_{3}^{n} & \text { in } \Omega, \\ \operatorname{div} \mathbf{e}^{n}=0 & \text { in } \Omega, \\ \mathbf{e}^{n}=0 & \text { on } \partial \Omega, \\ \mathbf{e}^{0}=0 & \text { in } \Omega .\end{cases}
$$

From (2.8), we can show that

$$
\begin{aligned}
& \left\|\mathbf{e}^{n}\right\|_{2}+\left\|p^{n}-P^{n}\right\|_{1} \\
\leq & C\left(\left\|D_{\tau} \mathbf{e}^{n}\right\|_{0}+\left\|\boldsymbol{\Lambda}_{1}^{n}\right\|_{0}+\left\|\boldsymbol{\Lambda}_{2}^{n}\right\|_{0}+\left\|\mathbf{R}_{1}^{n}\right\|_{0}+\left\|\mathbf{R}_{2}^{n}\right\|_{0}+\left\|\mathbf{R}_{3}^{n}\right\|_{0}\right),
\end{aligned}
$$

which together with (4.12)-(4.13b) yields

$$
\left\|\mathbf{e}^{n}\right\|_{2}+\left\|p^{n}-P^{n}\right\|_{1} \leq C\left(\left\|D_{\tau} \mathbf{e}^{n}\right\|_{0}+K_{0} \tau\right) .
$$

Summing (4.20) up from 1 to $n$ and using (4.16) result in

$$
\tau \sum_{m=1}^{n}\left\|\mathbf{e}^{m}\right\|_{2}^{2}+\tau \sum_{m=1}^{n}\left\|p^{n}-P^{n}\right\|_{1}^{2} \leq C K_{0}^{2} \tau^{2},
$$

which also leads to

$$
\left\|\mathbf{e}^{n}\right\|_{2}+\left\|p^{n}-P^{n}\right\|_{1} \leq C K_{0} \tau^{\frac{1}{2}} .
$$

On one hand, from (4.21), we find that

$$
\begin{aligned}
& \tau \sum_{m=1}^{n}\left\|D_{\tau} \mathbf{U}^{m}\right\|_{2}^{2} \leq 2 \tau \sum_{m=1}^{n}\left(\left\|D_{\tau} \mathbf{e}^{m}\right\|_{2}^{2}+\left\|D_{\tau} \mathbf{u}^{m}\right\|_{2}^{2}\right) \leq C K_{0}^{2}, \\
& \tau \sum_{m=1}^{n}\left\|D_{\tau} P^{m}\right\|_{1}^{2} \leq 2 \tau \sum_{m=1}^{n}\left(\left\|D_{\tau}\left(P^{m}-p^{m}\right)\right\|_{1}^{2}+\left\|D_{\tau} p^{m}\right\|_{1}^{2}\right) \leq C K_{0}^{2} .
\end{aligned}
$$

On the other hand, by (4.22) and assume that $\tau<\tau_{2}$, which ensures

$$
C K_{0} \tau^{\frac{1}{2}} \leq 1
$$

then we have

$$
\left\|\mathbf{e}^{n}\right\|_{2}+\left\|p^{n}-P^{n}\right\|_{1} \leq 1
$$


which also implies

$$
\begin{aligned}
& \left\|\mathbf{U}^{n}\right\|_{2} \leq\left\|\mathbf{e}^{n}\right\|_{2}+\left\|\mathbf{u}^{n}\right\|_{2} \leq 1+K \\
& \left\|P^{n}\right\|_{1} \leq\left\|P^{n}-p^{n}\right\|_{1}+\left\|p^{n}\right\|_{1} \leq 1+K .
\end{aligned}
$$

The mathematic induction is closed. Therefore, when $\tau<\tau^{*}=\min \left\{\tau_{1}, \tau_{2}\right\}$, (4.6a)-(4.6d) hold.

\subsection{The boundedness of $\mathrm{U}_{h}^{n}$}

In this section, we are devoted to derive the bounds of $\mathbf{U}_{h}^{n}$. First of all, the weak formulation of the problem (4.3) reads as

$$
\begin{cases}\left(D_{\tau} \mathbf{U}^{n}, \mathbf{v}\right)+v\left(\nabla \mathbf{U}^{n}, \nabla \mathbf{v}\right)-\left(\operatorname{div} \mathbf{v}, P^{n}\right)+b\left(\mathbf{U}^{n-1}, \mathbf{U}^{n}, \mathbf{v}\right) & \\ +\alpha\left(\left|\mathbf{U}^{n-1}\right|^{r-2} \mathbf{U}^{n-1}, \mathbf{v}\right)=\left(\mathbf{f}^{n}, \mathbf{v}\right), & \forall \mathbf{v} \in V, \\ \left(\operatorname{div} \mathbf{U}^{n}, q\right)=0, & \forall q \in Q, \\ \mathbf{U}^{0}=\mathbf{u}_{0} . & \end{cases}
$$

Let $\mathbf{e}_{h}^{n}=\mathbf{U}_{h}^{n}-I_{h} \mathbf{U}^{n}$, and subtract (4.2) from (4.27), then we obtain the error equations

$$
\begin{cases}\left(D_{\tau}\left(\mathbf{U}^{n}-I_{h} \mathbf{U}^{n}\right), \mathbf{v}_{h}\right)-\left(D_{\tau} \mathbf{e}_{h}^{n}, \mathbf{v}_{h}\right)-v\left(\nabla \mathbf{e}_{h}^{n}, \nabla \mathbf{v}_{h}\right)-\left(\operatorname{div} \mathbf{v}_{h}, J_{h} P^{n}-P_{h}^{n}\right) \\ \quad+\alpha\left(\left|\mathbf{U}^{n-1}\right|^{r-2} \mathbf{U}^{n-1}-\left|I_{h} \mathbf{U}^{n-1}\right|^{r-2} I_{h} \mathbf{U}^{n-1}, \mathbf{v}_{h}\right) & \\ \quad+\alpha\left(\left|I_{h} \mathbf{U}^{n-1}\right|^{r-2} I_{h} \mathbf{U}^{n-1}-\left|\mathbf{U}_{h}^{n-1}\right|^{r-2} \mathbf{U}_{h}^{n-1}, \mathbf{v}_{h}\right) & \\ +b\left(\mathbf{U}^{n-1}-I_{h} \mathbf{U}^{n-1} ; \mathbf{U}^{n}, \mathbf{v}_{h}\right)+b\left(I_{h} \mathbf{U}^{n-1} ; \mathbf{U}^{n}-I_{h} \mathbf{U}^{n}, \mathbf{v}_{h}\right) & \\ \quad-b\left(\mathbf{e}_{h}^{n-1} ; I_{h} \mathbf{U}^{n}, \mathbf{v}_{h}\right)-b\left(\mathbf{U}_{h}^{n-1} ; \mathbf{e}_{h}^{n}, \mathbf{v}_{h}\right)=0, & \forall \mathbf{v}_{h} \in V_{h}, \\ \left(\operatorname{div} \mathbf{e}_{h}^{n}, q_{h}\right)=0, & \forall q_{h} \in Q_{h} .\end{cases}
$$

Next we give the space error estimate and the bounds of $\mathbf{U}_{h}^{n}$.

Lemma 4.2. Let $\left(\mathbf{U}^{n}, P^{n}\right)$ and $\left(\mathbf{U}_{h}^{n}, P_{h}^{n}\right)$ be the solutions of the problems (4.27) and (4.2), respectively. Then under the conditions of Lemma 4.1 and assume that $h$ is small enough, we have

$$
\max _{1 \leq n \leq N}\left\|\mathbf{e}_{h}^{n}\right\|_{0} \leq C L_{4} h^{2}
$$

and

$$
\begin{aligned}
& \max _{1 \leq n \leq N}\left\|\mathbf{U}_{h}^{n}\right\|_{0, \infty} \leq C\left(1+\frac{K}{v}\right), \\
& \max _{1 \leq n \leq N}\left\|\nabla \mathbf{U}_{h}^{n}\right\|_{0,3} \leq C\left(1+\frac{K}{v}\right),
\end{aligned}
$$


where

$$
\begin{aligned}
L_{4}^{2}:=\exp & \left\{C\left(\alpha^{2} M^{2} \frac{(1+K)^{2 r-4}}{v^{2 r-3}}+\frac{(1+K)^{2}}{v^{3}}\right)\right\} \\
& \cdot\left(\frac{K_{0}^{2}}{v^{3}}+\alpha^{2} M^{2} \frac{(1+K)^{2 r-2}}{v^{2 r-1}}+\frac{(1+K)^{4}}{v^{3}}+\frac{(1+K)^{4}}{v^{5}}\right) .
\end{aligned}
$$

Proof. Now, we prove the following inequality

$$
\left\|\mathbf{e}_{h}^{m}\right\|_{0} \leq C L_{4} h^{2},
$$

by mathematical induction for all $m=0,1, \cdots, N$.

Since $\mathbf{e}_{h}^{0}=0$, (4.31) holds for $m=0$.

Assume that (4.31) holds for $m \leq n-1$, and $h$ is small enough to ensure that

$$
L_{4} h<1
$$

then by (3.3a), (4.6c) and the inverse inequality, we deduce

$$
\begin{aligned}
\left\|\mathbf{U}_{h}^{m}\right\|_{0, \infty} & \leq\left\|I_{h} \mathbf{U}^{m}\right\|_{0, \infty}+\left\|\mathbf{e}_{h}^{m}\right\|_{0, \infty} \leq C\left(\left\|\mathbf{U}^{m}\right\|_{2}+\frac{1}{v}\left\|P^{m}\right\|_{1}+h^{-1}\left\|\mathbf{e}_{h}^{m}\right\|_{0}\right) \\
& \leq C\left(1+\frac{K}{v}\right) .
\end{aligned}
$$

For $m=n$, taking $\mathbf{v}_{h}=\mathbf{e}_{h}^{n} \in V_{h}^{\operatorname{div}}$ in (4.28) yields

$$
\begin{aligned}
& \frac{1}{2 \tau}\left(\left\|\mathbf{e}_{h}^{n}\right\|_{0}^{2}+\left\|\mathbf{e}_{h}^{n}-\mathbf{e}_{h}^{n-1}\right\|_{0}^{2}-\left\|\mathbf{e}_{h}^{n-1}\right\|_{0}^{2}\right)+v\left\|\nabla \mathbf{e}_{h}^{n}\right\|_{0}^{2} \\
= & \left(D_{\tau}\left(\mathbf{U}^{n}-I_{h} \mathbf{U}^{n}\right), \mathbf{e}_{h}^{n}\right)+\alpha\left(\left|\mathbf{U}^{n-1}\right|^{r-2} \mathbf{U}^{n-1}-\left|I_{h} \mathbf{U}^{n-1}\right|^{r-2} I_{h} \mathbf{U}^{n-1}, \mathbf{e}_{h}^{n}\right) \\
& -\alpha\left(\left|\mathbf{U}_{h}^{n-1}\right|^{r-2} \mathbf{U}_{h}^{n-1}-\left|I_{h} \mathbf{U}^{n-1}\right|^{r-2} I_{h} \mathbf{U}^{n-1}, \mathbf{e}_{h}^{n}\right) \\
& +b\left(\mathbf{U}^{n-1}-I_{h} \mathbf{U}^{n-1} ; \mathbf{U}^{n}, \mathbf{e}_{h}^{n}\right)+b\left(I_{h} \mathbf{U}^{n-1} ; \mathbf{U}^{n}-I_{h} \mathbf{U}^{n}, \mathbf{e}_{h}^{n}\right) \\
& -b\left(\mathbf{e}_{h}^{n-1} ; I_{h} \mathbf{U}^{n}, \mathbf{e}_{h}^{n}\right):=\sum_{i=1}^{\Delta} G_{i} .
\end{aligned}
$$

Next we estimate all terms on the right hand-side of (4.34). From (3.3c), we find

$$
\begin{aligned}
\left|G_{1}\right| & \leq C h^{2}\left(\left\|D_{\tau} \mathbf{U}^{n}\right\|_{2}+\frac{1}{v}\left\|D_{\tau} P^{n}\right\|_{1}\right)\left\|\mathbf{e}_{h}^{n}\right\|_{0} \\
& \leq \frac{C}{v^{3}} h^{4}\left(\left\|D_{\tau} \mathbf{U}^{n}\right\|_{2}^{2}+\left\|D_{\tau} P^{n}\right\|_{1}^{2}\right)+\frac{v}{7}\left\|\nabla \mathbf{e}_{h}^{n}\right\|_{0}^{2} .
\end{aligned}
$$


Owing to Lemma 2.2, Lemma 3.1, and Lemma 4.1, the nonlinear damping terms can be bounded as

$$
\begin{aligned}
\left|G_{2}\right| & \leq \alpha M\left\|\mathbf{U}^{n-1}-I_{h} \mathbf{U}^{n-1}\right\|_{0}\left(\left\|\mathbf{U}^{n-1}\right\|_{0, \infty}+\left\|I_{h} \mathbf{U}^{n-1}\right\|_{0, \infty}\right)^{r-2}\left\|\mathbf{e}_{h}^{n}\right\|_{0} \\
& \leq C \alpha^{2} M^{2} \frac{(1+K)^{2 r-2}}{v^{2 r-1}} h^{4}+\frac{v}{7}\left\|\nabla \mathbf{e}_{h}^{n}\right\|_{0}^{2} \\
\left|G_{3}\right| & \leq \alpha M\left\|\mathbf{e}_{h}^{n-1}\right\|_{0}\left(\left\|\mathbf{U}_{h}^{n-1}\right\|_{0, \infty}+\left\|I_{h} \mathbf{U}^{n-1}\right\|_{0, \infty}\right)^{r-2}\left\|\mathbf{e}_{h}^{n}\right\|_{0} \\
& \leq C \alpha^{2} M^{2} \frac{(1+K)^{2 r-4}}{v^{2 r-3}}\left\|\mathbf{e}_{h}^{n-1}\right\|_{0}^{2}+\frac{v}{7}\left\|\nabla \mathbf{e}_{h}^{n}\right\|_{0}^{2} .
\end{aligned}
$$

By virtual of Lemma 2.1, Lemma 3.1, and Lemma 4.1, we can verify that

$$
\begin{aligned}
\left|G_{4}\right| & \leq C\left\|\mathbf{U}^{n-1}-I_{h} \mathbf{U}^{n-1}\right\|_{0}\left(\left\|\nabla \mathbf{U}^{n}\right\|_{0,3}+\left\|\mathbf{U}^{n}\right\|_{0, \infty}\right)\left\|\nabla \mathbf{e}_{h}^{n}\right\|_{0} \\
& \leq C \frac{(1+K)^{4}}{v^{3}} h^{4}+\frac{v}{7}\left\|\nabla \mathbf{e}_{h}^{n}\right\|_{0}^{2} \\
\left|G_{5}\right| & \leq C\left(\left\|I_{h} \mathbf{U}^{\mathbf{n}-1}\right\|_{0, \infty}+\left\|\nabla I_{h} \mathbf{U}^{n-1}\right\|_{0,3}\right)\left\|\nabla \mathbf{e}_{h}^{n}\right\|_{0}\left\|\mathbf{U}^{n}-I_{h} \mathbf{U}^{n}\right\|_{0} \\
& \leq C \frac{(1+K)^{4}}{v^{5}} h^{4}+\frac{v}{7}\left\|\nabla \mathbf{e}_{h}\right\|_{0}^{2}, \\
\left|G_{6}\right| & \leq C\left\|\mathbf{e}_{h}^{n-1}\right\|_{0}\left(\left\|\nabla I_{h} \mathbf{U}^{n}\right\|_{0,3}+\left\|I_{h} \mathbf{U}^{n}\right\|_{0, \infty}\right)\left\|\nabla \mathbf{e}_{h}^{n}\right\|_{0} \\
& \leq C \frac{(1+K)^{2}}{v^{3}}\left\|\mathbf{e}_{h}^{n-1}\right\|_{0}^{2}+\frac{v}{7}\left\|\nabla \mathbf{e}_{h}^{n}\right\|_{0}^{2} .
\end{aligned}
$$

Inserting (4.35)-(4.37c) into (4.34), we arrive at

$$
\begin{aligned}
& \frac{1}{2 \tau}\left(\left\|\mathbf{e}_{h}^{n}\right\|_{0}^{2}+\left\|\mathbf{e}_{h}^{n}-\mathbf{e}_{h}^{n-1}\right\|_{0}^{2}-\left\|\mathbf{e}_{h}^{n-1}\right\|_{0}^{2}\right)+\frac{v}{7}\left\|\nabla \mathbf{e}_{h}^{n}\right\|_{0}^{2} \\
\leq & C\left(\alpha^{2} M^{2} \frac{(1+K)^{2 r-4}}{v^{2 r-3}}+\frac{(1+K)^{2}}{v^{3}}\right)\left\|\mathbf{e}_{h}^{n-1}\right\|_{0}^{2}+\frac{C}{v^{3}} h^{4}\left(\left\|D_{\tau} \mathbf{U}^{n}\right\|_{2}^{2}+\left\|D_{\tau} P^{n}\right\|_{1}^{2}\right) \\
& +C\left(\alpha^{2} M^{2} \frac{(1+K)^{2 r-2}}{v^{2 r-1}}+\frac{(1+K)^{4}}{v^{3}}+\frac{(1+K)^{4}}{v^{5}}\right) h^{4} .
\end{aligned}
$$

Then summing (4.38) up from 1 to $n$, using (4.6d) and remembering $\mathbf{e}_{h}^{0}=0$, it follows that

$$
\begin{aligned}
& \left\|\mathbf{e}_{h}^{n}\right\|_{0}^{2}+v \tau \sum_{m=1}^{n}\left\|\nabla \mathbf{e}_{h}^{m}\right\|_{0}^{2} \\
\leq & C \tau\left(\alpha^{2} M^{2} \frac{(1+K)^{2 r-4}}{v^{2 r-3}}+\frac{(1+K)^{2}}{v^{3}}\right) \sum_{m=1}^{n}\left\|\mathbf{e}_{h}^{m-1}\right\|_{0}^{2} \\
& +C\left(\frac{K_{0}^{2}}{v^{3}}+\alpha^{2} M^{2} \frac{(1+K)^{2 r-2}}{v^{2 r-1}}+\frac{(1+K)^{4}}{v^{3}}+\frac{(1+K)^{4}}{v^{5}}\right) h^{4} .
\end{aligned}
$$


By Gronwall lemma, we obtain

$$
\left\|\mathbf{e}_{h}^{n}\right\|_{0}^{2}+v \tau \sum_{m=1}^{n}\left\|\nabla \mathbf{e}_{h}^{m}\right\|_{0}^{2} \leq C L_{4}^{2} h^{4}
$$

which implies (4.31) holds for $m=n$. The mathematic induction is closed, and we get (4.29) immediately.

Finally, assume that $h$ is small enough to ensure that

$$
L_{4} h^{\frac{2}{3}}<1,
$$

then using (3.3a), (3.3b), (4.6c), (4.29) and the inverse inequality yields

$$
\begin{aligned}
\max _{1 \leq n \leq N}\left\|\mathbf{U}_{h}^{n}\right\|_{0, \infty} & \leq \max _{1 \leq n \leq N}\left(\left\|I_{h} \mathbf{U}^{n}\right\|_{0, \infty}+\left\|\mathbf{e}_{h}^{n}\right\|_{0, \infty}\right) \\
& \leq C \max _{1 \leq n \leq N}\left(\left\|\mathbf{U}^{n}\right\|_{2}+\frac{1}{v}\left\|P^{n}\right\|_{1}+h^{-1}\left\|\mathbf{e}_{h}^{n}\right\|_{0}\right) \leq C\left(1+\frac{K}{v}\right), \\
\max _{1 \leq n \leq N}\left\|\nabla \mathbf{U}_{h}^{n}\right\|_{0,3} & \leq \max _{1 \leq n \leq N}\left(\left\|\nabla I_{h} \mathbf{U}^{n}\right\|_{0,3}+\left\|\nabla \mathbf{e}_{h}^{n}\right\|_{0,3}\right) \\
& \leq C \max _{1 \leq n \leq N}\left(\left\|\mathbf{U}^{n}\right\|_{2}+\frac{1}{v}\left\|P^{n}\right\|_{1}+h^{-\frac{4}{3}}\left\|\mathbf{e}_{h}^{n}\right\|_{0}\right) \leq C\left(1+\frac{K}{v}\right) .
\end{aligned}
$$

The proof is completed.

\subsection{The error estimates}

Let $\overline{\mathbf{e}}_{h}^{n}=\mathbf{U}_{h}^{n}-I_{h} \mathbf{u}^{n}$, and subtract (4.2) from (2.3) with $t=t_{n}$, then we have the following error equations

$$
\left\{\begin{array}{l}
\left(\mathbf{u}_{t}^{n}-D_{\tau} \mathbf{u}^{n}, \mathbf{v}_{h}\right)+\left(D_{\tau}\left(\mathbf{u}^{n}-I_{h} \mathbf{u}^{n}\right), \mathbf{v}_{h}\right)-\left(D_{\tau} \overline{\mathbf{e}}_{h}^{n}, \mathbf{v}_{h}\right)-v\left(\nabla \overline{\mathbf{e}}_{h}^{n}, \nabla \mathbf{v}_{h}\right) \\
\quad-\left(\operatorname{div} \mathbf{v}_{h}, J_{h} p^{n}-P_{h}^{n}\right)+\alpha\left(\left|\mathbf{u}^{n}\right|^{r-2} \mathbf{u}^{n}-\left|\mathbf{u}^{n-1}\right|^{r-2} \mathbf{u}^{n-1}, \mathbf{v}_{h}\right) \\
\quad+\alpha\left(\left|\mathbf{u}^{n-1}\right|^{r-2} \mathbf{u}^{n-1}-\left|I_{h} \mathbf{u}^{n-1}\right|^{r-2} I_{h} \mathbf{u}^{n-1}, \mathbf{v}_{h}\right) \\
\quad+\alpha\left(\left|I_{h} \mathbf{u}^{n-1}\right|^{r-2} I_{h} \mathbf{u}^{n-1}-\left|\mathbf{U}_{h}^{n-1}\right|^{r-2} \mathbf{U}_{h}^{n-1}, \mathbf{v}_{h}\right) \\
\quad+b\left(\mathbf{u}^{n}-\mathbf{u}^{n-1} ; \mathbf{u}^{n}, \mathbf{v}_{h}\right)+b\left(\mathbf{u}^{n-1}-I_{h} \mathbf{u}^{n-1} ; \mathbf{u}^{n}, \mathbf{v}_{h}\right) \\
\quad+b\left(I_{h} \mathbf{u}^{n-1} ; \mathbf{u}^{n}-I_{h} \mathbf{u}^{n}, \mathbf{v}_{h}\right)-b\left(\overline{\mathbf{e}}_{h}^{n-1} ; I_{h} \mathbf{u}^{n}, \mathbf{v}_{h}\right)-b\left(\mathbf{U}_{h}^{n-1} ; \overline{\mathbf{e}}_{h}^{n}, \mathbf{v}_{h}\right)=0, \\
\left(\operatorname{div} \overline{\mathbf{e}}_{h}^{n}, q_{h}\right)=0 .
\end{array}\right.
$$

Next based on the Lemma 4.2, we derive unconditional optimal error estimates of the fully discrete scheme (4.2). 
Theorem 4.1. Let $(\mathbf{u}, p)$ and $\left(\mathbf{U}_{h}^{n}, P_{h}^{n}\right)$ be the solutions of the problems (2.3) and (4.2), respectively. Under the conditions of Lemma 4.1, we have

$$
\max _{1 \leq n \leq N}\left\|\overline{\mathbf{e}}_{h}^{n}\right\|_{0} \leq C e^{C L_{5}^{2}}\left(L_{6} \tau+L_{7} h^{k+1}\right)
$$

where

$$
\begin{aligned}
& L_{5}^{2}:=\left(\alpha^{2} M^{2} \frac{(1+K)^{2 r-4}}{v^{2 r-3}}+\frac{K^{2}}{v^{3}}\right), \\
& L_{6}^{2}:=\frac{1}{v}\left(K^{2}+\alpha^{2} M^{2} K^{2 r-2}+K^{4}\right), \\
& L_{7}^{2}:=\left(\frac{K^{2}}{v^{3}}+\alpha^{2} M^{2} \frac{K^{2 r-2}}{v^{2 r-1}}+\frac{K^{4}}{v^{3}}+\frac{K^{4}}{v^{5}}\right) .
\end{aligned}
$$

Proof. Choosing $\mathbf{v}_{h}=\overline{\mathbf{e}}_{h}^{n} \in V_{h}^{\text {div }}$ in the first formula of (4.43) yields

$$
\begin{aligned}
& \frac{1}{2 \tau}\left(\left\|\overline{\mathbf{e}}_{h}^{n}\right\|_{0}^{2}+\left\|\overline{\mathbf{e}}_{h}^{n}-\overline{\mathbf{e}}_{h}^{n-1}\right\|_{0}^{2}-\left\|\overline{\mathbf{e}}_{h}^{n-1}\right\|_{0}^{2}\right)+v\left\|\nabla \overline{\mathbf{e}}_{h}^{n}\right\|_{0}^{2} \\
= & \left(\mathbf{u}_{t}^{n}-D_{\tau} \mathbf{u}^{n}, \overline{\mathbf{e}}_{h}^{n}\right)+\left(D_{\tau}\left(\mathbf{u}^{n}-I_{h} \mathbf{u}^{n}\right), \overline{\mathbf{e}}_{h}^{n}\right)+\alpha\left(\left|\mathbf{u}^{n}\right|^{r-2} \mathbf{u}^{n}-\left|\mathbf{u}^{n-1}\right|^{r-2} \mathbf{u}^{n-1}, \overline{\mathbf{e}}_{h}^{n}\right) \\
& +\alpha\left(\left|\mathbf{u}^{n-1}\right|^{r-2} \mathbf{u}^{n-1}-\left|I_{h} \mathbf{u}^{n-1}\right|^{r-2} I_{h} \mathbf{u}^{n-1}, \overline{\mathbf{e}}_{h}^{n}\right) \\
& +\alpha\left(\left|I_{h} \mathbf{u}^{n-1}\right|^{r-2} I_{h} \mathbf{u}^{n-1}-\left|\mathbf{U}_{h}^{n-1}\right|^{r-2} \mathbf{U}_{h}^{n-1}, \overline{\mathbf{e}}_{h}^{n}\right) \\
& +b\left(\mathbf{u}^{n}-\mathbf{u}^{n-1} ; \mathbf{u}^{n}, \overline{\mathbf{e}}_{h}^{n}\right)+b\left(\mathbf{u}^{n-1}-I_{h} \mathbf{u}^{n-1} ; \mathbf{u}^{n}, \overline{\mathbf{e}}_{h}^{n}\right) \\
& +b\left(I_{h} \mathbf{u}^{n-1} ; \mathbf{u}^{n}-I_{h} \mathbf{u}^{n}, \overline{\mathbf{e}}_{h}^{n}\right)-b\left(\overline{\mathbf{e}}_{h}^{n-1} ; I_{h} \mathbf{u}^{n}, \overline{\mathbf{e}}_{h}^{n}\right): \triangleq \sum_{i=1}^{9} S_{i} .
\end{aligned}
$$

Then we estimate the nine terms on the right hand-side of (4.45). By (4.11a) and (4.11b), we can easily derive the temporal error estimates

$$
\begin{aligned}
\left|S_{1}\right| & \leq\left\|\mathbf{u}_{t}^{n}-D_{\tau} \mathbf{u}^{n}\right\|_{0}\left\|\overline{\mathbf{e}}_{h}^{n}\right\|_{0} \leq C \frac{K^{2}}{v} \tau^{2}+\frac{v}{10}\left\|\nabla \overline{\mathbf{e}}_{h}^{n}\right\|_{0}^{2}, \\
\left|S_{3}\right| & \leq \alpha M\left\|\mathbf{u}^{n}-\mathbf{u}^{n-1}\right\|_{0}\left(\left\|\mathbf{u}^{n-1}\right\|_{0, \infty}+\left\|\mathbf{u}^{n}\right\|_{0, \infty}\right)^{r-2}\left\|\overline{\mathbf{e}}_{h}^{n}\right\|_{0} \\
& \leq C \alpha^{2} M^{2} \frac{K^{2 r-2}}{v} \tau^{2}+\frac{v}{10}\left\|\nabla \overline{\mathbf{e}}_{h}^{n}\right\|_{0}^{2}, \\
\left|S_{6}\right| & \leq C\left\|\nabla\left(\mathbf{u}^{n}-\mathbf{u}^{n-1}\right)\right\|_{0}\left\|\nabla \mathbf{u}^{n}\right\|_{0}\left\|\nabla \overline{\mathbf{e}}_{h}^{n}\right\|_{0} \leq C \frac{K^{4}}{v} \tau^{2}+\frac{v}{10}\left\|\nabla \overline{\mathbf{e}}_{h}^{n}\right\|_{0}^{2} .
\end{aligned}
$$


Using the same argument as that in Lemma 4.2, it gives the space error estimates

$$
\begin{gathered}
\left|S_{2}\right|+\left|S_{4}\right|+\left|S_{5}\right|+\left|S_{7}\right|+\left|S_{8}\right|+\left|S_{9}\right| \\
\leq C L_{5}^{2}\left\|\overline{\mathbf{e}}_{h}^{n-1}\right\|_{0}^{2}+C L_{7}^{2} h^{2 k+2}+\frac{6 v}{10}\left\|\nabla \overline{\mathbf{e}}_{h}^{n}\right\|_{0}^{2} .
\end{gathered}
$$

Substituting the above estimates into (4.45), it follows that

$$
\begin{aligned}
& \frac{1}{2 \tau}\left(\left\|\overline{\mathbf{e}}_{h}^{n}\right\|_{0}^{2}+\left\|\overline{\mathbf{e}}_{h}^{n}-\overline{\mathbf{e}}_{h}^{n-1}\right\|_{0}^{2}-\left\|\overline{\mathbf{e}}_{h}^{n-1}\right\|_{0}^{2}\right)+\frac{v}{10}\left\|\nabla \overline{\mathbf{e}}_{h}^{n}\right\|_{0}^{2} \\
\leq & C\left(L_{5}^{2}\left\|\overline{\mathbf{e}}_{h}^{n-1}\right\|_{0}^{2}+L_{6}^{2} \tau^{2}+L_{7}^{2} h^{2 k+2}\right) .
\end{aligned}
$$

Summing (4.48) up from 1 to $n$ and observing that $\overline{\mathbf{e}}_{h}^{0}=0$, we get

$$
\left\|\overline{\mathbf{e}}_{h}^{n}\right\|_{0}^{2}+v \tau \sum_{m=1}^{n}\left\|\nabla \overline{\mathbf{e}}_{h}^{m}\right\|_{0}^{2} \leq C\left(L_{5}^{2} \sum_{m=1}^{n}\left\|\overline{\mathbf{e}}_{h}^{m-1}\right\|_{0}^{2}+L_{6}^{2} \tau^{2}+L_{7}^{2} h^{2 k+2}\right),
$$

which together with Gronwall lemma yields (4.44) immediately.

Then we are in a position to derive the error estimate for $\left\|\nabla \overline{\mathbf{e}}_{h}^{n}\right\|_{0}$.

Theorem 4.2. Under the conditions of Theorem 4.1, we have

$$
\max _{1 \leq n \leq N}\left\|\nabla \overline{\mathbf{e}}_{h}^{n}\right\|_{0} \leq \frac{C}{v} e^{\frac{C}{v} L_{8}^{2}}\left(L_{9} \tau+L_{10} h^{k}\right),
$$

where

$$
\begin{aligned}
& L_{8}:=1+\frac{K}{v} \\
& L_{9}^{2}:=K^{2}+\alpha^{2} M^{2} K^{2 r-2}+K^{4}+\alpha^{2} M^{2} \frac{(1+K)^{2 r-4}}{v^{2 r-4}} e^{C L_{5}^{2}} L_{6}^{2} \\
& L_{10}^{2}:=\frac{K^{2}}{v^{2}}+\alpha^{2} M^{2} \frac{K^{2 r-2}}{v^{2 r-2}}+\frac{K^{4}}{v^{2}}+\frac{K^{4}}{v^{4}}+\alpha^{2} M^{2} \frac{(1+K)^{2 r-4}}{v^{2 r-4}} e^{C L_{5}^{2}} L_{7}^{2} .
\end{aligned}
$$

Proof. First, taking $\mathbf{v}_{h}=D_{\tau} \overline{\mathbf{e}}_{h}^{n} \in V_{h}^{\text {div }}$ in (4.43) results in

$$
\begin{aligned}
& \left\|D_{\tau} \overline{\mathbf{e}}_{h}^{n}\right\|_{0}^{2}+\frac{v}{2} D_{\tau}\left\|\nabla \overline{\mathbf{e}}_{h}^{n}\right\|_{0}^{2} \\
\leq & \left(\mathbf{u}_{t}^{n}-D_{\tau} \mathbf{u}^{n}, D_{\tau} \overline{\mathbf{e}}_{h}^{n}\right)+\left(D_{\tau}\left(\mathbf{u}^{n}-I_{h} \mathbf{u}^{n}\right), D_{\tau} \overline{\mathbf{e}}_{h}^{n}\right) \\
& +\alpha\left(\left|\mathbf{u}^{n}\right|^{r-2} \mathbf{u}^{n}-\left|\mathbf{u}^{n-1}\right|^{r-2} \mathbf{u}^{n-1}, D_{\tau} \overline{\mathbf{e}}_{h}^{n}\right) \\
& +\alpha\left(\left|\mathbf{u}^{n-1}\right|^{r-2} \mathbf{u}^{n-1}-\left|I_{h} \mathbf{u}^{n-1}\right|^{r-2} I_{h} \mathbf{u}^{n-1}, D_{\tau} \overline{\mathbf{e}}_{h}^{n}\right)
\end{aligned}
$$




$$
\begin{aligned}
& +\alpha\left(\left|I_{h} \mathbf{u}^{n-1}\right|^{r-2} I_{h} \mathbf{u}^{n-1}-\left|\mathbf{U}_{h}^{n-1}\right|^{r-2} \mathbf{U}_{h}^{n-1}, D_{\tau} \overline{\mathbf{e}}_{h}^{n}\right) \\
& +b\left(\mathbf{u}^{n}-\mathbf{u}^{n-1} ; \mathbf{u}^{n}, D_{\tau} \overline{\mathbf{e}}_{h}^{n}\right)+b\left(\mathbf{u}^{n-1}-I_{h} \mathbf{u}^{n-1} ; \mathbf{u}^{n}, D_{\tau} \overline{\mathbf{e}}_{h}^{n}\right) \\
& +b\left(I_{h} \mathbf{u}^{n-1} ; \mathbf{u}^{n}-I_{h} \mathbf{u}^{n}, D_{\tau} \overline{\mathbf{e}}_{h}^{n}\right)-b\left(\overline{\mathbf{e}}_{h}^{n-1} ; I_{h} \mathbf{u}^{n}, D_{\tau} \overline{\mathbf{e}}_{h}^{n}\right) \\
& -b\left(\mathbf{U}_{h}^{n-1} ; \overline{\mathbf{e}}_{h}^{n}, D_{\tau} \overline{\mathbf{e}}_{h}^{n}\right): \triangleq \sum_{i=1}^{10} T_{i} .
\end{aligned}
$$

Based on Lemmas 2.1, 2.2, 3.1, 4.2, and Theorem 4.1, it is not difficult to check that

$$
\begin{gathered}
\left\|D_{\tau} \overline{\mathbf{e}}_{h}^{n}\right\|_{0}^{2}+v \frac{\left\|\nabla \overline{\mathbf{e}}_{h}^{n}\right\|_{0}^{2}-\left\|\nabla \overline{\mathbf{e}}_{h}^{n-1}\right\|_{0}^{2}}{\tau} \\
\leq C L_{8}^{2}\left(\left\|\nabla \overline{\mathbf{e}}_{h}^{n-1}\right\|_{0}^{2}+\left\|\nabla \overline{\mathbf{e}}_{h}^{n}\right\|_{0}^{2}\right)+C L_{9}^{2} \tau^{2}+C L_{10}^{2} h^{2 k} .
\end{gathered}
$$

Summing (4.53) up from 1 to $n$ gives

$$
\tau \sum_{m=1}^{n}\left\|D_{\tau} \overline{\mathbf{e}}_{h}^{m}\right\|_{0}^{2}+v\left\|\nabla \overline{\mathbf{e}}_{h}^{n}\right\|_{0}^{2} \leq C\left(\frac{L_{8}^{2}}{v} \tau \sum_{m=1}^{n} v\left\|\nabla \overline{\mathbf{e}}_{h}^{m}\right\|_{0}^{2}+L_{9}^{2} \tau^{2}+L_{10}^{2} h^{2 k}\right) .
$$

Consequently, by Gronwall lemma, there exists $\tau_{3}>0$ such that when $\tau \leq \tau_{3}$, it gives

$$
\tau \sum_{m=1}^{n}\left\|D_{\tau} \overline{\mathbf{e}}_{h}^{m}\right\|_{0}^{2}+v\left\|\nabla \overline{\mathbf{e}}_{h}^{n}\right\|_{0}^{2} \leq C e^{\frac{C}{v} L_{8}^{2}}\left(L_{9}^{2} \tau^{2}+L_{10}^{2} h^{2 k}\right),
$$

which implies the desired result.

Now it remains to derive the error estimate of the pressure. Similar to that of the semi-discrete case, we first estimate $\left\|D_{\tau} \overline{\mathbf{e}}_{h}^{n}\right\|_{-1}$.

Lemma 4.3. Under the conditions of Theorem 4.1, we have

$$
\max _{1 \leq n \leq N}\left\|D_{\tau} \overline{\mathbf{e}}_{h}^{n}\right\|_{-1} \leq \frac{C}{v^{2}}(1+K) e^{\frac{C}{v} L_{8}^{2}}\left(L_{9} \tau+L_{10} h^{k}\right) .
$$

Proof. Choosing $\mathbf{v}_{h}=A_{h}^{-1} D_{\tau} \overline{\mathbf{e}}_{h}^{n} \in V_{h}^{\text {div }}$ in the first formula of (4.43) yields

$$
\begin{aligned}
& \left\|A_{h}^{-\frac{1}{2}} D_{\tau} \overline{\mathbf{e}}_{h}^{n}\right\|_{0}^{2}=\left(D_{\tau} \overline{\mathbf{e}}_{h}^{n}, A_{h}^{-1} D_{\tau} \overline{\mathbf{e}}_{h}^{n}\right) \\
= & \left(\mathbf{u}_{t}^{n}-D_{\tau} \mathbf{u}^{n}, A_{h}^{-1} D_{\tau} \overline{\mathbf{e}}_{h}^{n}\right)+\left(D_{\tau}\left(\mathbf{u}^{n}-I_{h} \mathbf{u}^{n}\right), A_{h}^{-1} D_{\tau} \overline{\mathbf{e}}_{h}^{n}\right) \\
& -v\left(\nabla \overline{\mathbf{e}}_{h}^{n}, \nabla A_{h}^{-1} D_{\tau} \overline{\mathbf{e}}_{h}^{n}\right)+\alpha\left(\left|\mathbf{u}^{n}\right|^{r-2} \mathbf{u}^{n}-\left|\mathbf{u}^{n-1}\right|^{r-2} \mathbf{u}^{n-1}, A_{h}^{-1} D_{\tau} \overline{\mathbf{e}}_{h}^{n}\right) \\
& +\alpha\left(\left|\mathbf{u}^{n-1}\right|^{r-2} \mathbf{u}^{n-1}-\left|I_{h} \mathbf{u}^{n-1}\right|^{r-2} I_{h} \mathbf{u}^{n-1}, A_{h}^{-1} D_{\tau} \overline{\mathbf{e}}_{h}^{n}\right)
\end{aligned}
$$




$$
\begin{aligned}
& +\alpha\left(\left|I_{h} \mathbf{u}^{n-1}\right|^{r-2} I_{h} \mathbf{u}^{n-1}-\left|\mathbf{U}_{h}^{n-1}\right|^{r-2} \mathbf{U}_{h}^{n-1}, A_{h}^{-1} D_{\tau} \overline{\mathbf{e}}_{h}^{n}\right) \\
& +b\left(\mathbf{u}^{n}-\mathbf{u}^{n-1} ; \mathbf{u}^{n}, A_{h}^{-1} D_{\tau} \overline{\mathbf{e}}_{h}^{n}\right)+b\left(\mathbf{u}^{n-1}-I_{h} \mathbf{u}^{n-1} ; \mathbf{u}^{n}, A_{h}^{-1} D_{\tau} \overline{\mathbf{e}}_{h}^{n}\right) \\
& +b\left(I_{h} \mathbf{u}^{n-1} ; \mathbf{u}^{n}-I_{h} \mathbf{u}^{n}, A_{h}^{-1} D_{\tau} \overline{\mathbf{e}}_{h}^{n}\right)-b\left(\overline{\mathbf{e}}_{h}^{n-1} ; I_{h} \mathbf{u}^{n}, A_{h}^{-1} D_{\tau} \overline{\mathbf{e}}_{h}^{n}\right) \\
& -b\left(\mathbf{U}_{h}^{n-1} ; \overline{\mathbf{e}}_{h}^{n}, A_{h}^{-1} D_{\tau} \overline{\mathbf{e}}_{h}^{n}\right) .
\end{aligned}
$$

Based on Theorems 4.1 and 4.2, and using the similar argument as that in Lemma 3.3 yield the desired result.

With the aid of Lemma 4.3 and the inf-sup condition, it is easy to derive the error estimate for the pressure.

Theorem 4.3. Under the conditions of Theorem 4.1, we have

$$
\max _{1 \leq n \leq N}\left\|J_{h} p^{n}-P_{h}^{n}\right\|_{0} \leq \frac{C}{v^{2}}(1+K) e^{\frac{C}{v} L_{8}^{2}}\left(L_{9} \tau+L_{10} h^{k}\right) .
$$

Finally, from Theorems 4.1-4.3, and the triangle inequality, we deduce the following error estimates.

Theorem 4.4. Under the conditions of Theorem 4.1, we have

$$
\begin{aligned}
& \max _{1 \leq n \leq N}\left\|\mathbf{u}^{n}-\mathbf{U}_{h}^{n}\right\|_{0} \leq C e^{C L_{5}^{2}}\left(L_{6} \tau+L_{7} h^{k}\right), \\
& \max _{1 \leq n \leq N}\left(\left\|\nabla\left(\mathbf{u}^{n}-\mathbf{U}_{h}^{n}\right)\right\|_{0}\right) \leq \frac{C}{v} e^{\frac{C}{v} L_{8}^{2}}\left(L_{9} \tau+L_{10} h^{k}\right), \\
& \max _{1 \leq n \leq N}\left(\left\|p^{n}-P_{h}^{n}\right\|_{0}\right) \leq \frac{C}{v^{2}}(1+K) e^{C L_{8}^{2}}\left(L_{9} \tau+L_{10} h^{k}\right) .
\end{aligned}
$$

\section{Numerical implementation}

In this section, we present two numerical examples to illustrate the theoretical analysis. We take $\Omega=[0,1] \times[0,1], r=3, \alpha=1$ for the two examples.

Example 5.1. Consider the example with the following exact solutions

$$
\begin{aligned}
& u_{1}=x^{2}(x-1)^{2} y(y-1)(2 y-2) e^{-t}, \\
& u_{2}=-x(x-1) y^{2}(y-1)^{2}(2 x-2) e^{-t}, \\
& p=\left(x^{2}-y^{2}\right) e^{-t} .
\end{aligned}
$$

Then the source term $\mathbf{f}$ is determined by Eqs. (1.1) with the above prescribed velocity and pressure.

The lowest-order Hood-Taylor element pairs, the triangular $P_{2}-P_{1}$ element pair and rectangular $Q_{2}-Q_{1}$ element pair [43], are used. The triangle meshes are obtained from 
Table 1: The numerical results for the $P_{2}-P_{1}$ pair at $t=0.5$ with $\tau=8 h^{3}$.

\begin{tabular}{||ccccccc||}
\hline & $\left\|\mathbf{u}^{n}-\mathbf{U}_{h}^{n}\right\|_{0}$ & Order & $\left\|\nabla\left(\mathbf{u}^{n}-\mathbf{U}_{h}^{n}\right)\right\|_{0}$ & Order & $\left\|p^{n}-P_{h}^{n}\right\|_{0}$ & Order \\
\hline $4 \times 4$ & $6.01239 \mathrm{E}-5$ & & $3.73547 \mathrm{E}-3$ & & $4.94167 \mathrm{E}-3$ & \\
$8 \times 8$ & $7.12100 \mathrm{E}-6$ & 3.07779 & $1.00856 \mathrm{E}-3$ & 1.88899 & $1.22546 \mathrm{E}-3$ & 2.01168 \\
$16 \times 16$ & $8.28573 \mathrm{E}-7$ & 3.10338 & $2.57977 \mathrm{E}-4$ & 1.96698 & $3.05930 \mathrm{E}-4$ & 2.00205 \\
$32 \times 32$ & $1.00939 \mathrm{E}-7$ & 3.03715 & $6.49078 \mathrm{E}-5$ & 1.99078 & $7.64692 \mathrm{E}-5$ & 2.00025 \\
\hline
\end{tabular}

Table 2: The numerical results for the $P_{2}-P_{1}$ pair at $t=1$ with $\tau=8 h^{3}$.

\begin{tabular}{||lllllll||}
\hline & $\left\|\mathbf{u}^{n}-\mathbf{U}_{h}^{n}\right\|_{0}$ & Order & $\left\|\nabla\left(\mathbf{u}^{n}-\mathbf{U}_{h}^{n}\right)\right\|_{0}$ & Order & $\left\|p^{n}-P_{h}^{n}\right\|_{0}$ & Order \\
\hline $4 \times 4$ & $3.61934 \mathrm{E}-5$ & & $2.26596 \mathrm{E}-3$ & & $2.99743 \mathrm{E}-3$ & \\
$8 \times 8$ & $4.31891 \mathrm{E}-6$ & 3.06699 & $6.11723 \mathrm{E}-4$ & 1.88917 & $7.43277 \mathrm{E}-4$ & 2.01175 \\
$16 \times 16$ & $5.02544 \mathrm{E}-7$ & 3.10334 & $1.56471 \mathrm{E}-4$ & 1.96698 & $1.85556 \mathrm{E}-4$ & 2.00205 \\
$32 \times 32$ & $6.12222 \mathrm{E}-8$ & 3.03712 & $3.93686 \mathrm{E}-5$ & 1.99078 & $4.63809 \mathrm{E}-5$ & 2.00025 \\
\hline
\end{tabular}

Table 3: The numerical results for the $Q_{2}-Q_{1}$ pair at $t=0.5$ with $\tau=8 h^{3}$.

\begin{tabular}{||ccccccc||}
\hline & $\left\|\mathbf{u}^{n}-\mathbf{U}_{h}^{n}\right\|_{0}$ & Order & $\left\|\nabla\left(\mathbf{u}^{n}-\mathbf{U}_{h}^{n}\right)\right\|_{0}$ & Order & $\left\|p^{n}-P_{h}^{n}\right\|_{0}$ & Order \\
\hline $4 \times 4$ & $5.11023 \mathrm{E}-5$ & & $1.35802 \mathrm{E}-3$ & & $3.99666 \mathrm{E}-3$ & \\
$8 \times 8$ & $6.49058 \mathrm{E}-6$ & 2.97697 & $3.38218 \mathrm{E}-4$ & 2.00548 & $9.98989 \mathrm{E}-4$ & 2.00026 \\
$16 \times 16$ & $8.14136 \mathrm{E}-7$ & 2.99500 & $8.44598 \mathrm{E}-5$ & 2.00162 & $2.49742 \mathrm{E}-4$ & 2.00003 \\
$32 \times 32$ & $1.01867 \mathrm{E}-7$ & 2.99858 & $2.11090 \mathrm{E}-5$ & 2.00041 & $6.24355 \mathrm{E}-5$ & 2.00000 \\
\hline
\end{tabular}

Table 4: The numerical results for the $Q_{2}-Q_{1}$ pair at $t=1$ with $\tau=8 h^{3}$.

\begin{tabular}{||ccccccc||}
\hline & $\left\|\mathbf{u}^{n}-\mathbf{U}_{h}^{n}\right\|_{0}$ & Order & $\left\|\nabla\left(\mathbf{u}^{n}-\mathbf{U}_{h}^{n}\right)\right\|_{0}$ & Order & $\left\|p^{n}-P_{h}^{n}\right\|_{0}$ & Order \\
\hline $4 \times 4$ & $3.09883 \mathrm{E}-5$ & & $8.23743 \mathrm{E}-4$ & & $2.42412 \mathrm{E}-3$ & \\
$8 \times 8$ & $3.93676 \mathrm{E}-6$ & 2.97664 & $2.05140 \mathrm{E}-4$ & 2.00559 & $6.05917 \mathrm{E}-4$ & 2.00027 \\
$16 \times 16$ & $4.93804 \mathrm{E}-7$ & 2.99500 & $5.12275 \mathrm{E}-5$ & 2.00162 & $1.51476 \mathrm{E}-4$ & 2.00003 \\
$32 \times 32$ & $6.17863 \mathrm{E}-8$ & 2.99858 & $1.28033 \mathrm{E}-5$ & 2.00041 & $3.78690 \mathrm{E}-5$ & 2.00000 \\
\hline
\end{tabular}

the uniform rectangular meshes by inserting diagonal edges. To confirm our theoretical analysis, we take $\tau=8 h^{3}$, then

$$
\max _{1 \leq n \leq N}\left(\left\|\mathbf{u}^{n}-\mathbf{U}_{h}^{n}\right\|_{0}+h\left\|\nabla\left(\mathbf{u}^{n}-\mathbf{U}_{h}^{n}\right)\right\|_{0}+h\left\|p^{n}-P_{h}^{n}\right\|_{0}\right) \leq C h^{3} .
$$

The errors and convergence orders of the two element pairs with $v=1$ are showed in Tables 1-4. The errors $\left\|\mathbf{u}^{n}-\mathbf{U}_{h}^{n}\right\|_{0^{\prime}}\left\|\nabla\left(\mathbf{u}^{n}-\mathbf{U}_{h}^{n}\right)\right\|_{0}$ and $\left\|p^{n}-P_{h}^{n}\right\|_{0}$ are convergent at rates of $\mathcal{O}\left(h^{3}\right), \mathcal{O}\left(h^{2}\right)$ and $\mathcal{O}\left(h^{2}\right)$, respectively. It is clear that all the error estimates are optimal, which accord with our theoretical analysis completely.

To show the unconditional convergence of the scheme (4.2), we use several different time steps with a fixed $h=\frac{1}{16}$ at $t=1.0$. The errors are given in Tables 5-7. It can be seen that for the fixed $h$, when the time step increases gradually, the three errors $\left\|\mathbf{u}^{n}-\mathbf{U}_{h}^{n}\right\|_{0^{\prime}}$ 
Table 5: The errors of $\left\|\mathbf{u}^{n}-\mathbf{U}_{h}^{n}\right\|_{0}$ at $t=1$ with $h=\frac{1}{16}$ and $\tau=k h^{3}$.

\begin{tabular}{||ccccc||}
\hline & $k=2$ & $k=4$ & $k=8$ & $k=16$ \\
\hline$P_{2}-P_{1}$ pair & $5.04653 \mathrm{E}-7$ & $5.03862 \mathrm{E}-7$ & $5.02544 \mathrm{E}-7$ & $5.00982 \mathrm{E}-7$ \\
$Q_{2}-Q_{1}$ pair & $4.93417 \mathrm{E}-7$ & $4.93455 \mathrm{E}-7$ & $4.93804 \mathrm{E}-7$ & $4.95599 \mathrm{E}-7$ \\
\hline
\end{tabular}

Table 6: The errors of $\left\|\nabla\left(\mathbf{u}^{n}-\mathbf{U}_{h}^{n}\right)\right\|_{0}$ at $t=1$ with $h=\frac{1}{16}$ and $\tau=k h^{3}$.

\begin{tabular}{||ccccc||}
\hline & $k=2$ & $k=4$ & $k=8$ & $k=16$ \\
\hline$P_{2}-P_{1}$ pair & $1.56471 \mathrm{E}-04$ & $1.56471 \mathrm{E}-04$ & $1.56471 \mathrm{E}-04$ & $1.56472 \mathrm{E}-04$ \\
$Q_{2}-Q_{1}$ pair & $5.12271 \mathrm{E}-05$ & $5.12272 \mathrm{E}-05$ & $5.12275 \mathrm{E}-05$ & $5.12286 \mathrm{E}-05$ \\
\hline
\end{tabular}

Table 7: The errors of $\left\|p^{n}-P_{h}^{n}\right\|_{0}$ at $t=1$ with $h=\frac{1}{16}$ and $\tau=k h^{3}$.

\begin{tabular}{||ccccc||}
\hline & $k=2$ & $k=4$ & $k=8$ & $k=16$ \\
\hline$P_{2}-P_{1}$ pair & $1.85555 \mathrm{E}-04$ & $1.85555 \mathrm{E}-04$ & $1.85556 \mathrm{E}-04$ & $1.85556 \mathrm{E}-04$ \\
$Q_{2}-Q_{1}$ pair & $1.51476 \mathrm{E}-04$ & $1.51476 \mathrm{E}-04$ & $1.51476 \mathrm{E}-04$ & $1.51477 \mathrm{E}-04$ \\
\hline
\end{tabular}

$\left\|\nabla\left(\mathbf{u}^{n}-\mathbf{U}_{h}^{n}\right)\right\|_{0}$ and $\left\|p^{n}-P_{h}^{n}\right\|_{0}$ of the two pairs tend to be a constant, which implies that the time-restriction is not necessary.

Example 5.2. Consider the lid-driven cavity flow problem. We impose the normal component of the velocity to be zero on $\partial \Omega$ and the tangential component to be zero except along the top boundary where it is set to one.

In the computation, we use the $Q_{2}-Q_{1}$ element and take $v=1,0.1,0.01$. Three different time steps $\tau=0.1,0.01$ and 0.001 with a fixed $h=\frac{1}{20}$ at $t=1.0$ are tested. The numerical results along the horizontal and vertical centerline of the cavity are shown in Figs. 1-3. From these Figures, we can see that almost the same numerical results can be obtained for the three time steps, which also implies that our scheme (4.2) has excellent stability with the large time step.

\section{Acknowledgements}

This work was supported by Fundamental Research Funds for the Henan Provincial Colleges and Universities (No. 20A110002).

\section{References}

[1] D. BRESCH, AND B. DESJARDINS, Existence of global weak solutions for a 2D viscous shallow water equations and convergence to the quasi-geostrophic model, Commun. Math. Phys., 238(1-2) (2003), pp. 211-223. 

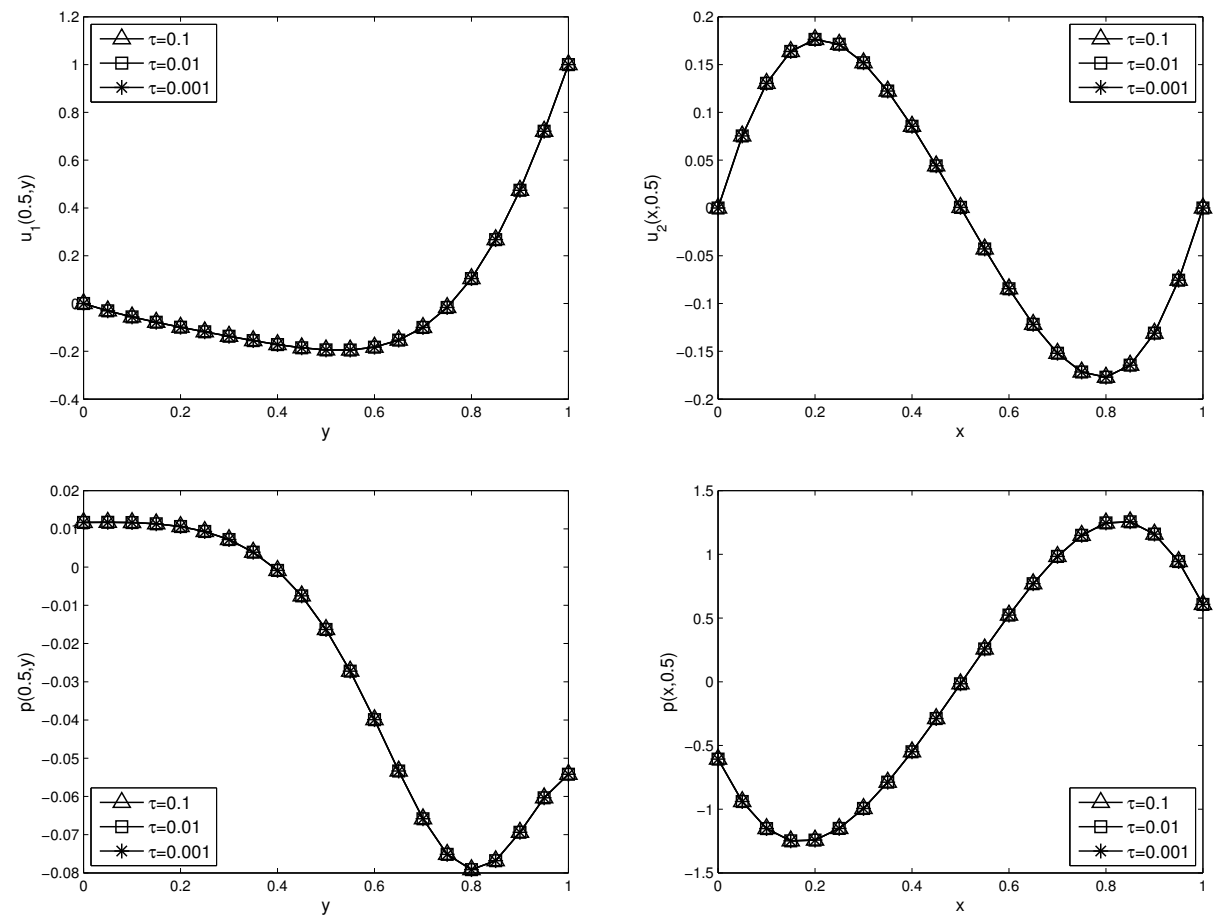

Figure 1: Comparisons of the velocity and pressure with $v=1$.

[2] D. BRESCH, B. DESJARDINS, AND C. LIN, On some compressible fluid models: Korteweg, lubrication, and shallow water systems, Commun. Part. Diff. Eq., 28(3-4) (2003), pp. 843-868.

[3] X. CAI, AND Q. JIU, Weak and strong solutions for the incompressible Navier-Stokes equations with damping term, J. Math. Anal. Appl., 343(2) (2008), pp. 799-809.

[4] Z. ZHANG, X. WU, AND M. LU, On the uniqueness of strong solution to the incompressible Navier-Stokes equations with damping, J. Math. Anal. Appl., 377(1) (2011), pp. 414-419.

[5] Y. SUN, AND H. RUI, MAC finite difference scheme for Stokes equations with damping on nonuniform grids, Comput. Math. Appl., 75(4) (2018), pp. 1272-1287.

[6] D. LIU, AND K. LI, Finite element analysis of the Stokes equations with damping, Math. Numer. Sin., 32(4) (2010), pp. 433-448.

[7] D. SHI, AND Z. YU, Superclose and superconvergence of finite element discretizations for the Stokes equations with damping, Appl. Math. Comput., 219(14) (2013), pp. 7693-7698.

[8] J. WU, P. HuANG, AND X. FENG, Numerical study on several stabilized finite element methods for the steady incompressible flow problem with damping, J. Appl. Math., (2013) 2013.

[9] M. LI, D. SHI, AND Y. DAI, Stabilized low order finite elements for Stokes equations with damping, J. Math. Anal. Appl., 435(1) (2016), pp. 646-660.

[10] Z. LI, D. SHI, AND M. LI, Stabilized mixed finite element methods for the Navier-Stokes equations with damping, Math. Method Appl. Sci., 42(2) (2019), pp. 605-619.

[11] M. LI, D. SHI, Z. LI, AND H. CHEN, Two-level mixed finite element methods for the NavierStokes equations with damping, J. Math. Anal. Appl., 470(1) (2019), pp. 292-307.

[12] H. QIU, AND L. MEI, Multi-level stabilized algorithms for the stationary incompressible Navier- 

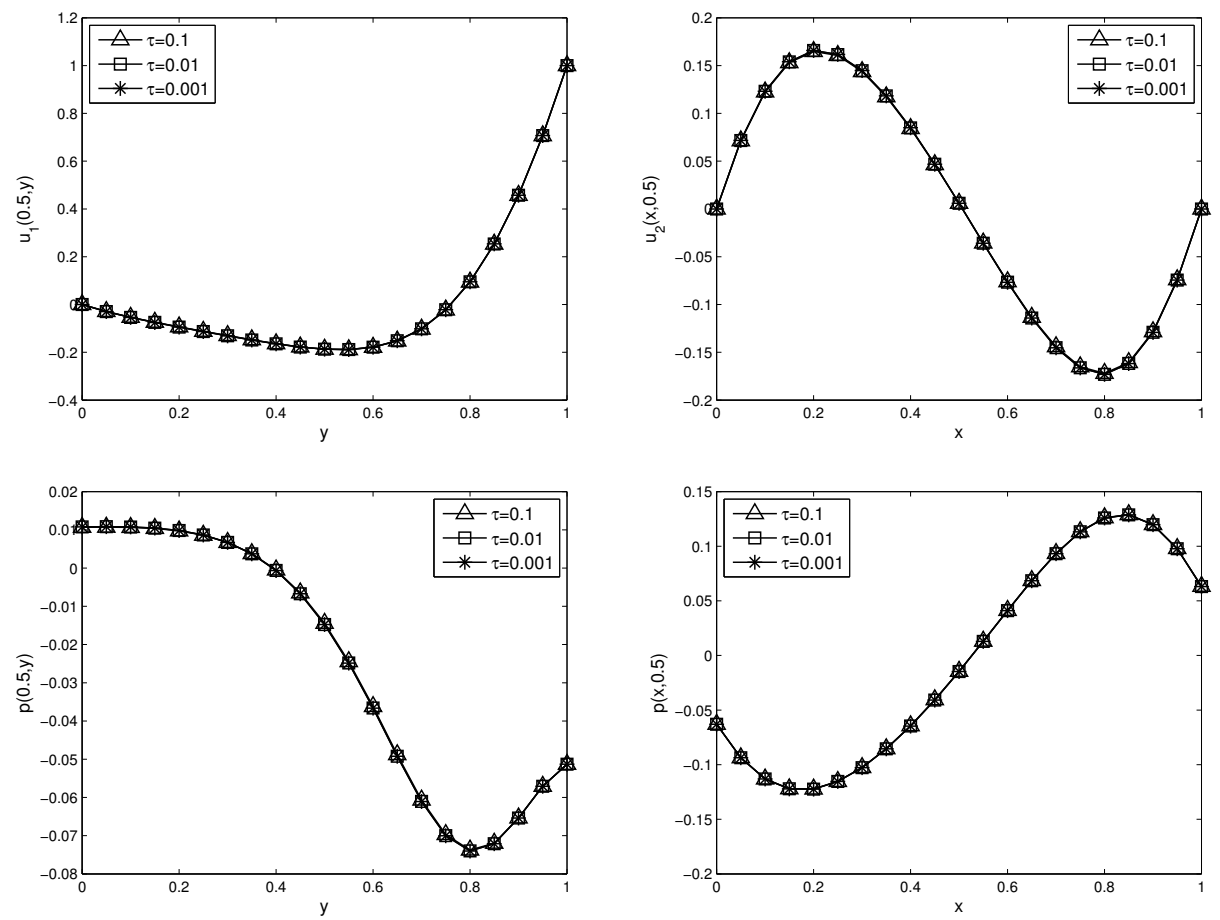

Figure 2: Comparisons of the velocity and pressure with $v=0.1$.

Stokes equations with damping, Appl. Numer. Math., 143 (2019), pp. 188-202.

[13] H. QIU, Y. ZHANG, AND L. MEI, A mixed-FEM for Navier-Stokes type variational inequality with nonlinear damping term, Comput. Math. Appl., 73(10) (2017), pp. 2191-2207.

[14] B. LI, AND W. SUN, Error analysis of linearized semi-implicit Galerkin finite element methods for nonlinear parabolic equations, Int. J. Numer. Anal. Mod., 10(3) (2013), pp. 622-633.

[15] B. LI, AND W. SUN, Unconditional convergence and optimal error estimates of a Galerkin-mixed FEM for incompressible miscible flow in porous media, SIAM J. Numer. Anal., 51(4) (2013), pp. 1959-1977.

[16] D. LI, AND J. WANG, Unconditionally optimal error analysis of Crank-Nicolson Galerkin FEMs for a strongly nonlinear parabolic system, J. Sci. Comput., 72(2) (2017), pp. 892-915.

[17] D. SHI, AND H. YANG, Unconditional optimal error estimates of a two-grid method for semilinear parabolic equation, Appl. Math. Comput., 310(1) (2017), pp. 40-47.

[18] D. SHI, AND J. WANG, Unconditional superconvergence analysis of conforming finite element for nonlinear parabolic equation, Appl. Math. Comput., 294(1) (2017), pp. 216-226.

[19] D. SHI, AND J. WANG, Unconditional superconvergence analysis for nonlinear hyperbolic equation with nonconforming finite element, Appl. Math. Comput., 305(15) (2017), pp. 1-16.

[20] J. WANG, A new error analysis of Crank-Nicolson Galerkin FEMs for a generalized nonlinear Schrödinger equation, J. Sci. Comput., 60(2) (2014), pp. 390-407.

[21] W. CAI, J. LI, AND Z. CHEN, Unconditional convergence and optimal error estimates of the Euler semi-implicit scheme for a generalized nonlinear Schrödinger equation, Adv. Comput. Math., 42(6) (2016), pp. 1311-1330. 

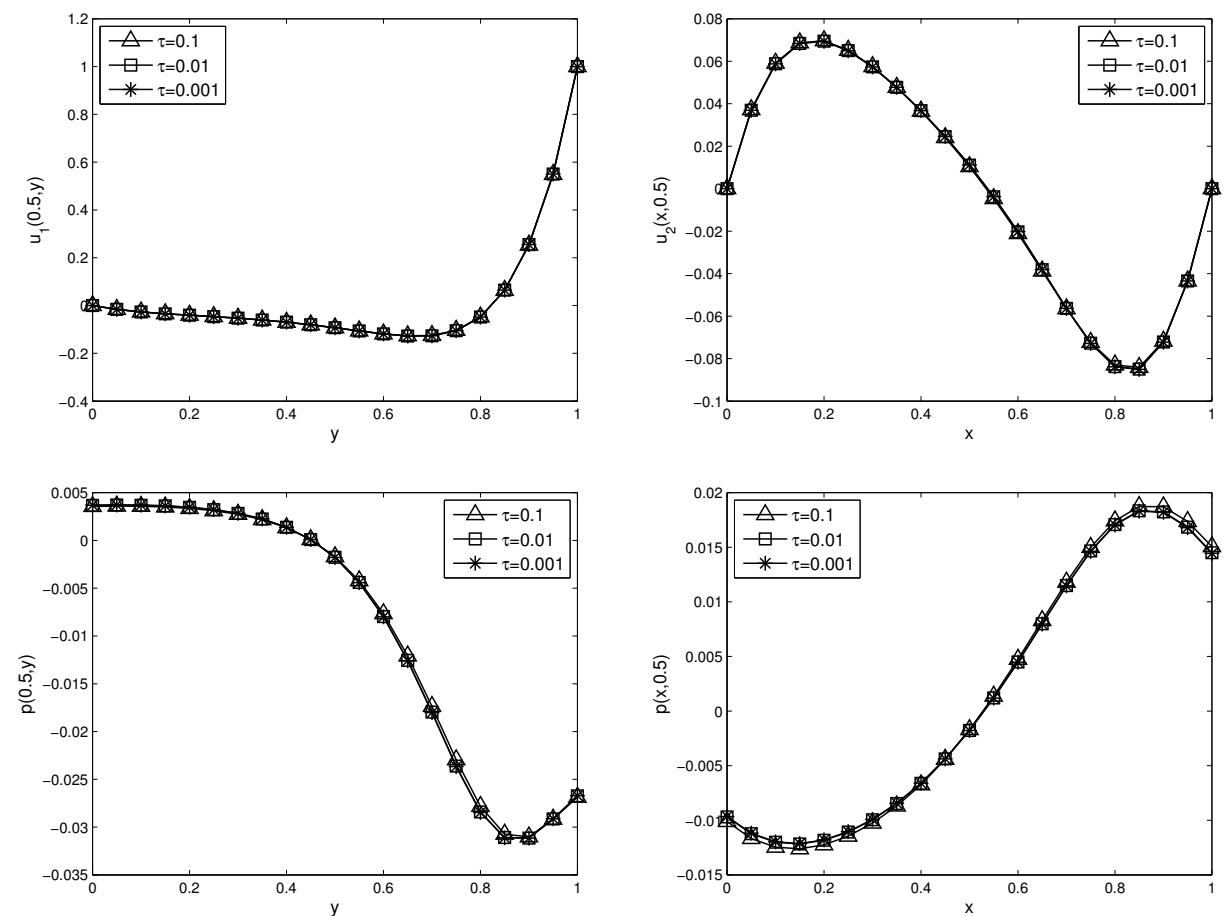

Figure 3: Comparisons of the velocity and pressure with $v=0.01$.

[22] W. CAI, J. LI, AND Z. CHEN, Unconditional optimal error estimates for BDF2-FEM for a nonlinear Schrödinger equation, J. Comput. Appl. Math., 331(15) (2018), pp. 23-41.

[23] D. SHI, AND H. YANG, Unconditionally optimal error estimates of a new mixed FEM for nonlinear Schrödinger equations, Adv. Comput. Math., 45(5-6) (2019), pp. 3173-3194.

[24] D. SHI, AND J. WANG, Unconditional superconvergence analysis of a Crank-Nicolson Galerkin FEM for nonlinear Schrödinger equation, J. Sci. Comput., 72(3) (2017), pp. 1093-1118.

[25] R. AN, Optimal error estimates of linearized Crank-Nicolson Galerkin method for Landau-Lifshitz equation, J. Sci. Comput., 69(1) (2016), pp. 1-27.

[26] C. YANG, A linearized Crank-Nicolson-Galerkin FEM for the time-dependent Ginzburg-Landau equations under the temporal gauge, Numer. Meth. Part. D. E., 30(4) (2014), pp. 1279-1290.

[27] D. SHI, AND Q. LIU, Unconditional superconvergent analysis of a linearized finite element method for Ginzburg-Landau equation, Appl. Numer. Math., 147 (2020), pp. 118-128.

[28] M. LI, D. SHI, AND J. WANG ET AL., Unconditional superconvergence analysis of the conservative linearized Galerkin FEMs for nonlinear Klein-Gordon-Schrödinger equation, Appl. Numer. Math., 142 (2019), pp. 47-63.

[29] B. LI, H. GAO, AND W. SUN, Unconditionally optimal error estimates of a Crank-Nicolson Galerkin method for the nonlinear thermistor equations, SIAM J. Numer. Anal., 52(2) (2014), pp. 933-954.

[30] H. GAO, Unconditional optimal error estimates of BDF-Galerkin FEMs for nonlinear thermistor equations, J. Sci. Comput., 66(2) (2016), pp. 504-527.

[31] H. GAO, B. LI, AND W. SUN, Stability and convergence of fully discrete Galerkin FEMs for the 
nonlinear thermistor equations in a nonconvex polygon, Numer. Math., 136(2) (2017), pp. 383409.

[32] Z. SI, J. WANG, AND W. SUN, Unconditional stability and error estimates of modified characteristics FEMs for the Navier-Stokes equations, Numer. Math., 134(1) (2016), pp. 139-161.

[33] H. ZHENG, J. YU, AND S. LI, Unconditional error estimates for time dependent viscoelastic fluid flow, Appl. Numer. Math., 119 (2017), pp. 1-17.

[34] Y. YANG, AND Z. SI, Unconditional stability and error estimates of the modified characteristics FEMs for the time-dependent incompressible MHD equations, Comput. Math. Appl., 77(1) (2019), pp. 263-283.

[35] J. HEYWOOD, AND R. RANNACHER, Finite-element approximation of the nonstationary NavierStokes problem. Part IV: Error analysis for second-order time discretization, SIAM J. Numer. Anal., 27(2) (1990), pp. 353-384.

[36] Y. HE, The Euler implicit/explicit scheme for the 2D time-dependent Navier-Stokes equations with smooth or non-smooth initial data, Math. Comput., 77(264) (2008), pp. 2097-2124.

[37] P. Ciarlet, The Finite Element Method for Elliptic Problems, North-Holland, Amsterdam, 1978.

[38] J. HEYWOOD, AND R. RANNACHER, Finite element approximation of the nonstationary NavierStokes problem. I. Regularity of solutions and second-order error estimates for spatial discretization, SIAM J. Numer. Anal., 19(2) (1982), pp. 275-311.

[39] V. Girault, R. NOCHETTO, AND R. SCOTT, Maximum-norm stability of the finite element Stokes projection, J. Math. Pures Appl., 84(3) (2005), pp. 279-330.

[40] V. JOHN, Finite Element Methods for Incompressible Flow Problems, Springer-Verlag, Berlin, 2016.

[41] J. De Frutos, B. Garcia-Archilla, V. John, AND J. Novo, Analysis of the grad-div stabilization for the time-dependent Navier-Stokes equations with inf-sup stable finite elements, Adv. Comput. Math., 44(1) (2018), pp. 195-225.

[42] B. Ayuso, B. Garcia-Archilla, AND J. Novo, The postprocessed mixed finite-element method for the Navier-Stokes equations, SIAM J. Numer. Anal., 43(3) (2005), pp. 1091-1111.

[43] D. Boffi, F. BreZZI, AND M. ForTin, Mixed Finite Element Methods and Applications, Springer, Heidelberg, 2013. 